\title{
Reconstruction et modernisation de la SNCF : les nouvelles rotondes pour locomotives à vapeur (1944-1952)
}

Reconstruction and modernization of the SNCF: the new rotundas for steam engines (1944-1952)

\section{Nicolas Nogue}

\section{OpenEdition \\ Journals}

Édition électronique

URL : https://journals.openedition.org/rhcf/1821

DOI : 10.4000/rhcf.1821

\section{Éditeur}

Rails \& histoire

\section{Édition imprimée}

Date de publication : 1 décembre 2003

Pagination : $510-546$

ISBN : 0996-9403

ISSN : 0996-9403

Référence électronique

Nicolas Nogue, "Reconstruction et modernisation de la SNCF : les nouvelles rotondes pour locomotives à vapeur (1944-1952) », Revue d'histoire des chemins de fer [En ligne], 28-29 | 2003, mis en ligne le 06 janvier 2015, consulté le 22 avril 2022. URL : http://journals.openedition.org/rhcf/1821 ; DOl : https://doi.org/10.4000/rhcf.1821 


\section{Reconstruction et modernisation de la SNCF : les nouvelle rotondes pour locomotives à vapeur (1944-1952)}

Après la Deuxième Guerre mondiale, la $\mathrm{SNCF}$ a fait appel à l'ingénieur-conseil Bernard Laffaille (1900-1955) pour la reconstruction et la modernisation de certains de ses équipements : rotondes pour locomotives à vapeur, ateliers d'entretien et de réparation du matériel roulant et autres « halles à trafic accéléré » abritant les nouvelles méthodes mécanisées de transbordement des colis ${ }^{1}$. De toutes les études proposées par Laffaille, seules les rotondes ont été intégralement réalisées selon ses conceptions ${ }^{2}$. Dès l'achèvement de la première d'entre elles, en 1946, à Avignon, les nouvelles remises ont été repérées et publiées par les revues spécialisées, notamment L'Architecture d'aujourd'hui (fig. 1 et fig. 2), et sont aujourd'hui répertoriées par les historiens parmi les principales réussites architecturales de la reconstruction française $e^{3}$. La rotonde à locomotives d'Avignon est ainsi inscrite à l'inventaire supplémentaire des monuments historiques depuis 1984.

Ces édifices font date dans le contexte de la reconstruction en raison, certes, de l'élégance de leurs lignes mais surtout par la modernité des méthodes de conception adoptées et des solutions constructives appliquées : élaboration de projets-types modulaires (fig. 3), mise en œuvre par préfabrication lourde, emploi de structures d'avant-garde (coques de béton armé) alors que, au même moment, on reconstruit la plupart des villes et des campagnes selon des techniques traditionnelles. Le chantier des rotondes SNCF constitue ainsi un véritable laboratoire technique et architectural dans la France de l'après-guerre, un laboratoire qui inaugure des méthodes ensuite généralisées, pour les opérations

1- Il s'agit des halles d'Angers-Saint-Laud, Lyon-Guillotière, Pantin-Bobigny et ParisTolbiac.

2- Les autres projets ont été amendés soit par les services de la SNCF, d'autres ingénieurs-conseils ou les entreprises adjudicataires. Seuls l'atelier accolé à la rotonde d'Avignon et la première tranche de la halle de Lyon ont été exécutés selon les plans de Laffaille. Les autres projets reprennent en général l'emploi de ses trumeaux en $\mathrm{V}$ pour les façades mais optent pour d'autres systèmes de couverture que ceux préconisés par l'ingénieur.

3- Voir: Gérard Monnier, L'Architecture en France, une histoire critique, 1918-1950, Paris, Philippe Sers éditeur, 1990, p. 378. 


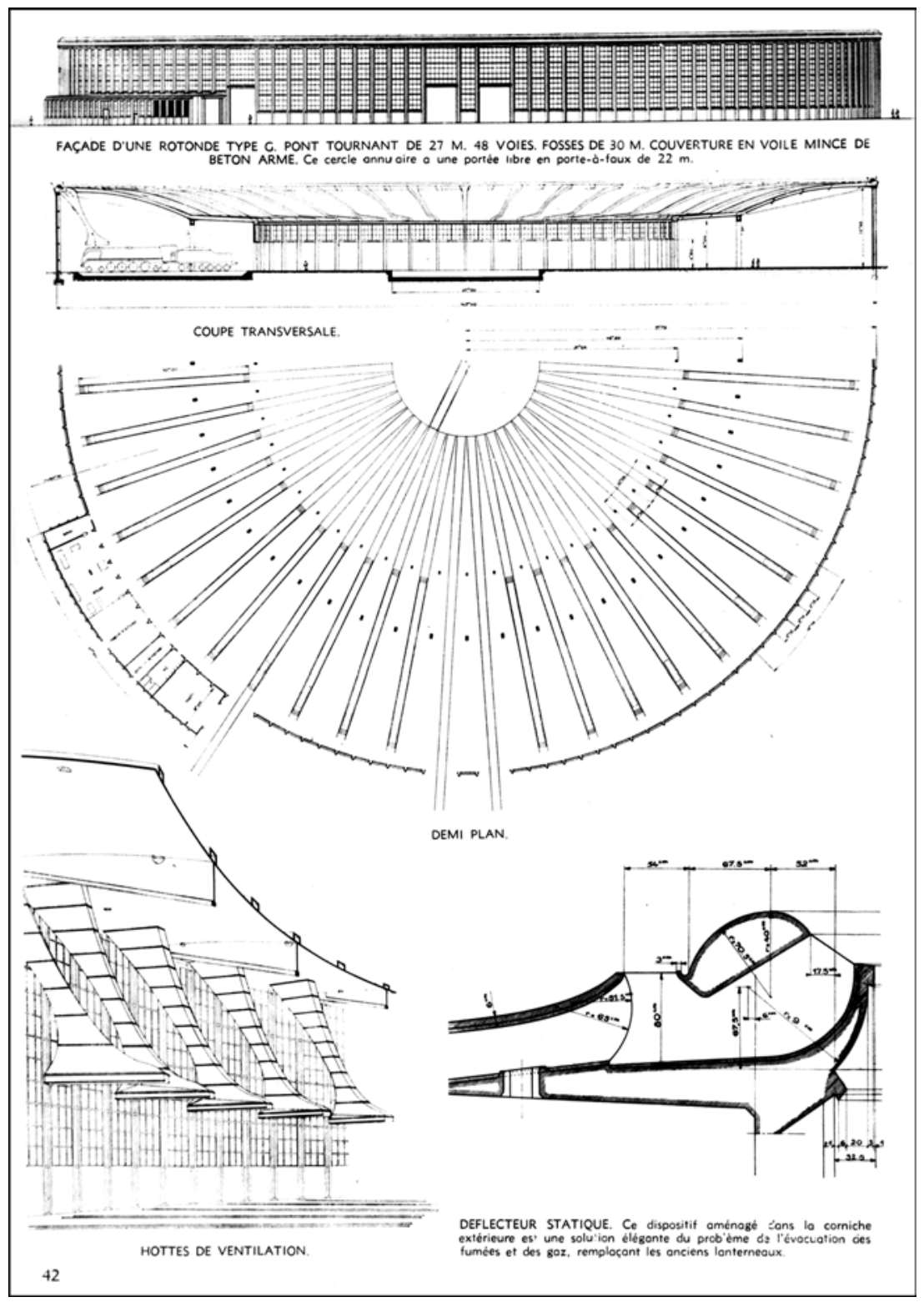

Figure 1. Illustration du premier article sur les rotondes SNCF de Laffaille, publié dans L'Architecture d'aujourd'hui, n 9 , novembre-décembre 1946. Élévation, coupe, plan et détails de la rotonde type G. Cl. N. Nogue. 


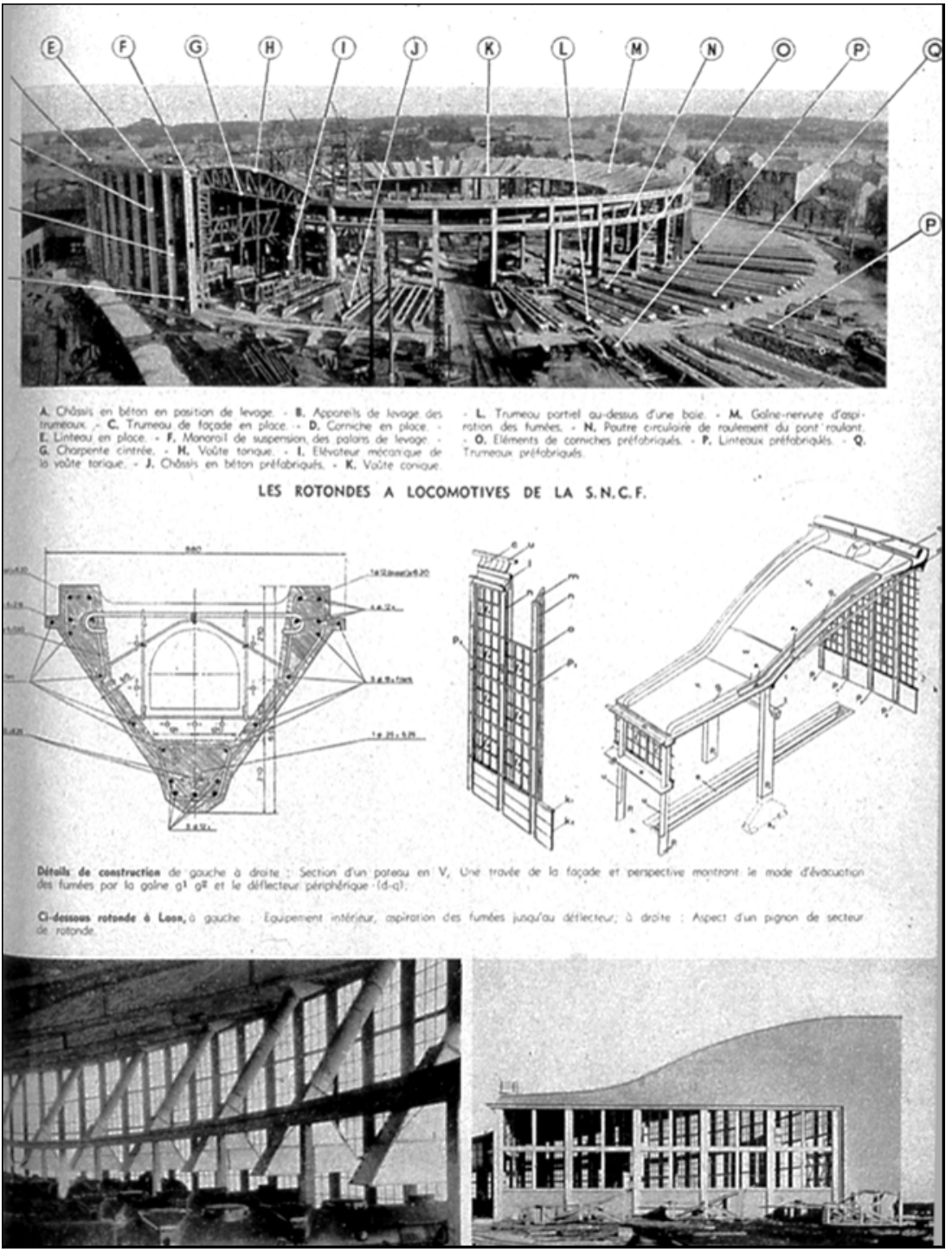

Figure 2. Illustration dans L'Architecture d'aujourd'hui, $\mathrm{n}^{\circ} 17,1948$. Vue du chantier de la rotonde d'Avignon, éléments types et deux vues d'une autre remise que celle $d^{\prime}$ Avignon (rotonde de Laon ?). Cl. N. Nogue. 
des grands ensembles de logements notamment. On n'en possédait pourtant qu'une connaissance très biaisée et incomplète puisque principalement véhiculée par sa médiatisation. Grâce à la découverte du fonds d'archives de l'ingénieur Bernard Laffaille et à son inventaire, cette lacune a toutefois pu être comblée et l'ensemble de l'opération reconstitué dans toute son envergure et sa complexitét.

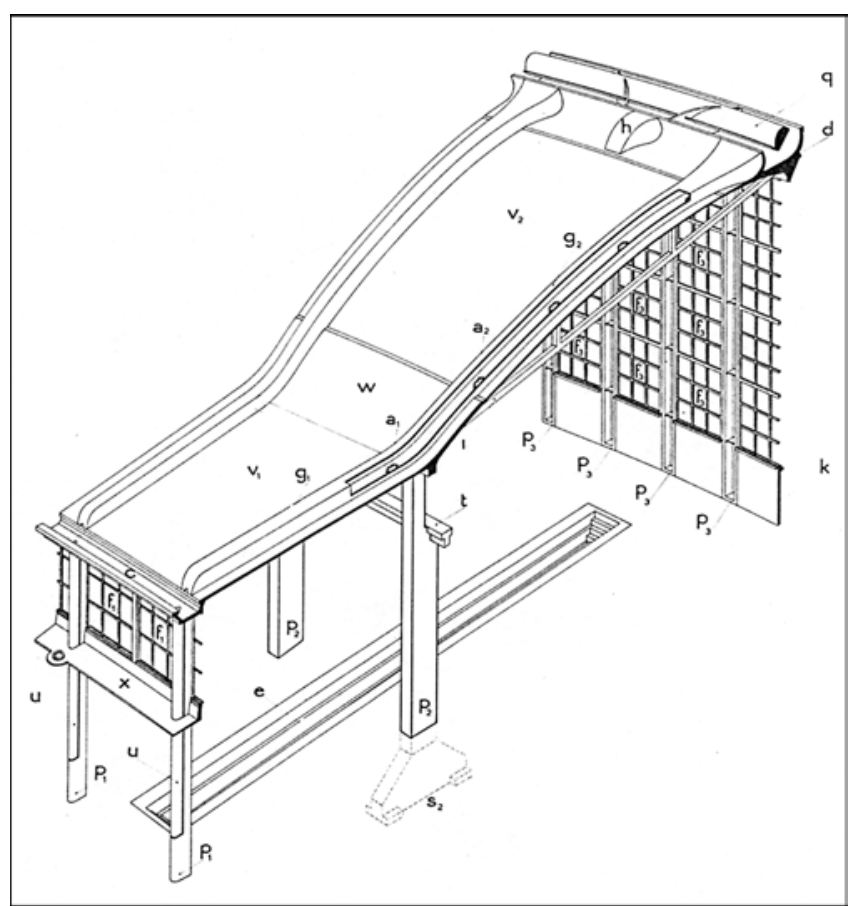

Figure 3. Travée-type « $P$ » ou « $G$ », ca 1945. Coll. SNCF, cl. N. Nogue.

4- J'ai retrouvé le fonds d'archives de l'ingénieur en 1993 et l'ai classé entre 1994 et 1999 à l'Institut français d'architecture (IFA). Son inventaire est publié dans : "Archives d'ingénieurs, Bernard Laffaille », Colonnes, $\mathrm{n}^{\circ}$ 18, IFA, Paris, mai 2002, p. 21-46. Le présent article est tiré de ma thèse de doctorat en histoire de l'art sur l'œuvre de Laffaille : Bernard Laffaille (1900-1955), ingénieur. De l'entreprise au bureau d'études : modes d'exercice et pensée technique, Université Paris I-Panthéon-Sorbonne, 2001. La première synthèse de mon étude sur l'œuvre de Laffaille pour la SNCF a été publiée dans l'article suivant : «La reconversion des rotondes SNCF conçues par Bernard Laffaille » in « Le patrimoine pour quoi faire ?", actes du colloque national du CILAC à Trégastel (5-6-7 octobre 1994), L'Archéologie industrielle en France, revue du CILAC, 1996, p. 43-48. 


\section{La commande}

Aucun document ne permet de cerner les circonstances exactes de la commande adressée par la SNCF à Laffaille. Toutefois, tout porte à croire que l'ingénieur a pu bénéficier de ses appuis politiques pour l'obtenir : le rôle qu'il a joué au sein de la Résistance - il a accueilli à son domicile une séance du Conseil national de la résistance - et ses relations entretenues, depuis lors, avec Georges Bidault et Claudius-Petit ont vraisemblablement été à l'origine de la collaboration de l'ingénieurconseil avec la SNCF.

Quoi qu'il en soit, les relations entre Laffaille et l'entreprise nationale débutent en octobre-novembre 1944 : date de l'élaboration et de la signature de son contrat. Son activité s'est exercée en tant qu'ingénieur-conseil de l'entreprise en liaison avec la « division des Bâtiments ", dirigée par Paul Peirani, et la « division des Ouvrages d'art », dirigée par Roger Vallette, services regroupés au sein de la «division centrale des Installations fixes et de la Construction ", elle-même dirigée par MM. Porchez puis Robert Lévi. La collaboration avec la division des Bâtiments a porté sur la conception architecturale des projets tandis que les questions purement techniques de calcul des structures et de résistance des matériaux ont été traitées avec la division des Ouvrages d'art et, le plus souvent, directement avec son responsable, R. Vallette.

Bien que les nombreux articles parus dans la presse spécialisée au sujet des rotondes ne mentionnent que rarement le nom de Bernard Laffaille, il est clair que l'ingénieur a conçu ces édifices - même si, bien sûr, les différents services de la SNCF sont intervenus au cours de l'élaboration des projets. Une note dactylographiée, intitulée « mode de fonctionnement des études de Bâtiment ", l'indique d'ailleurs ${ }^{5}$; elle décrit, dans un tableau, les phases que suit l'élaboration de tout projet et mentionne, pour chaque phase, l'intervention des différents acteurs en jeu : la SNCF, Bernard Laffaille, son bureau d'études ('Institut d'études techniques et professionnelles - l'IETP), l'entreprise adjudicataire, éventuellement un autre bureau d'études techniques. Or, pour toutes les étapes de la conception, ainsi que la vérification des dossiers d'exécution des entreprises, Laffaille intervient directement (soit personnellement, soit par l'intermédiaire de l'IETP).

En ce qui concerne les halles et ateliers, les archives montrent clairement que les services de la SNCF interviennent peu dans la conception même des projets : c'est Laffaille qui élabore l'intégralité de

5- Voir archives IFA 28/1. 
la solution structurale en réponse au programme que lui soumet la Société nationale. En revanche, pour les rotondes, on ne dispose pas d'archives concernant la conception des prototypes et sur lesquelles on puisse se baser pour prouver le rôle créateur de l'ingénieur. Il ne fait cependant pas de doute : E. Claudius-Petit, dans l'allocution qu'il prononce à l'Assemblée nationale en 1948, désigne Laffaille comme le concepteur de ces édifices ${ }^{6}$. L'ingénieur, dans une lettre au ministre de la Reconstruction et de l'Urbanisme, se présente bien lui-même comme « ingénieur conseil-maitre d'œuvre » des ouvrages réalisés pour la $\mathrm{SNCF}^{7}$.

Ces précisions ont leur importance car elles permettent de relativiser la contribution à la conception des rotondes de Paul Peirani, ingénieur polytechnicien, architecte D.P.L.G. et chef de la division des Bâtiments. En effet, si le premier article présentant ces édifices mentionne bien Bernard Laffaille comme leur concepteur, les articles suivants les attribuent à « P. Peirani, architecte et B. Laffaille, ingénieur ${ }^{8} »$. Tirant profit de sa position professionnelle forte et du prestige du titre d'architecte, il semble que Peirani ait cherché à s'attribuer, sinon la paternité, du moins un rôle significatif dans l'élaboration des rotondes et, du même coup, à cantonner Laffaille dans le rôle de l'ingénieur qui les a calculées. Or, en tout état de cause, on peut même s'interroger sur une quelconque participation directe du responsable de la division des Bâtiments dans la conception des remises à locomotives, dans la mesure où il apparaît très rarement dans la correspondance de Laffaille et parce qu'il n'a pas laissé, au sein même de la SNCF, le souvenir d'avoir joué un rôle créateur dans la mise au point de ces édifices?

6- Allocution prononcée le 27 août 1948, document conservé par la famille Laffaille. 7- IFA cote 37/4, lettre du 14 août 1948, de Laffaille à Claudius-Petit. Par ailleurs, suite à un accident grave intervenu le 24 avril 1947 sur le chantier de la rotonde de Creil, l'architecte Odent est chargé de mener une enquête pour en déterminer les causes. Laffaille, en tant que concepteur du projet, est convoqué à comparaitre en personne le 25 juin 1947 au tribunal de Creil. Le 11 juin 1947, il écrit à l'architecte : « Je vous précise que j'ai été l'auteur des projets des rotondes pour le compte de la SNCF. » (IFA, dossier cote 12/2).

8- Le premier article présentant les rotondes parait dans L'Architecture d'aujourd'bui, $\mathrm{n}^{\circ}$ 9, décembre 1946.

9- La correspondance de Laffaille montre clairement que le principal interlocuteur de l'ingénieur à la SNCF, lors de l'édification des rotondes, est R. Vallette, chef de la division des Ouvrages d'art. Par ailleurs, c'est M. Poupardin, alors architecte à la division des Bâtiments, qui me communiqua (entrevue du 27 mai 1991) l'information selon laquelle P. Peirani n'était pas considéré à la SNCF comme le concepteur des rotondes. 


\section{La conception d'ouvrages-types}

Pour la reconstruction des remises à locomotives à vapeur, le Service central (division des Bâtiments) a préféré le parti architectural et technique des rotondes à celui des dépôts rectangulaires, mettant ainsi fin à une controverse qui, depuis la fin du XIX ${ }^{e}$ siècle, voyait s'affronter les partisans de l'une et de l'autre solution. On a opté pour les rotondes dans la mesure où elles réduisent les surfaces d'emprise nécessaires et permettent une évolution plus rapide des machines. La guerre, de plus, avait montré que les ponts tournants constituent un dispositif moins fragile qu'on ne l'avait jusqu'alors pensé ${ }^{10}$. Les nouvelles remises s'inscrivent ainsi dans une lignée historique d'ouvrages de même typologie - notamment les célèbres rotondes PLM de l'entre-deux-guerres ouvrages dont elles améliorent sensiblement les performances fonctionnelles ${ }^{11}$.

Soulignons toutefois que dans certaines régions, qui ne souhaitaient pas se soumettre aux décisions de la direction parisienne de l'entreprise, des « remises rectangulaires » ont été édifiées entre 1945 et 1948, notamment dans les régions est et nord de la SNCF, comme à Châlons-sur-Marne (fig. 4), Nancy et Chaumont.

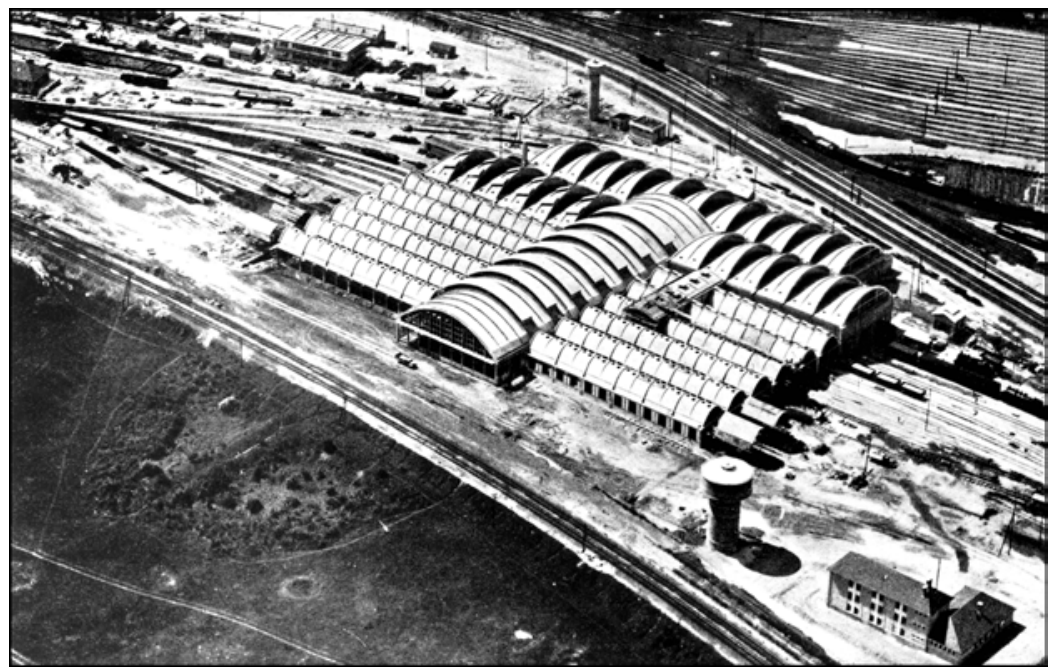

Figure 4. Remise rectangulaire de Châlons-sur-Marne (1945-1948). Coll. et cl. SNCF.

10- R. Gonon, "Reconstruction de dépôts à rotondes sur la région du Nord de la SNCF », Travaux, n 167 bis, supplément: "SNCF 1944-1948», p. 57-62.

11- Voir à ce sujet : Robert Lévi, « Nouvelles rotondes à machines de la SNCF », Revue générale des chemins de fer, $\mathrm{n}^{\circ} 1$, janvier 1948, p. 1-4. 
À l'instar des rotondes de Laffaille, les nouveaux édifices présentent des couvertures en voile mince de béton armé, notamment des coques cylindriques, paraboliques et conoïdes ${ }^{12}$.

\section{De fortes contraintes d'usage et d'économie}

La rotonde-type doit satisfaire à quatre contraintes essentielles : assurer une bonne évacuation des fumées produites par les locomotives, offrir un éclairage naturel aussi abondant que possible de manière à faciliter les travaux de réparation, présenter une structure assez flexible pour s'adapter, sans études ou travaux supplémentaires, aux besoins locaux en remisage et, enfin, dans un contexte de pénurie en matières premières, main-d'œuvre et financements, permettre l'économie et la rapidité maximales de la mise en œuvre des matériaux.

Ce dernier critère explique le choix du béton armé comme unique matériau constructif employé, au détriment du métal, du bois et de la maçonnerie. Le béton armé concentre, en effet, plusieurs avantages décisifs : il résiste bien aux fumées corrosives des machines, il souffre moins de pénurie que les autres matériaux, il offre la possibilité de préfabriquer le maximum d'éléments standardisés. Et, enfin, en cette période d'après-guerre, le savoir-faire et l'équipement que nécessite l'édification des rotondes existe encore dans le domaine de la mise en œuvre du béton armé, aussi bien au niveau de la main-d'œuvre qu'au niveau des entreprises de construction, ce qui n'est pas le cas pour la construction des charpentes en bois, par exemple.

\section{Une structure modulaire}

La solution constructive élaborée par Laffaille présente en plan la forme d'un anneau constitué de 48 modules identiques, chaque module abritant une fosse d'entretien. Un pont tournant, destiné à aiguiller les locomotives dans les modules, occupe le centre, non couvert, de l'édifice qui se compose de trois parties distinctes : les fondations (et les fosses de réparation), les poteaux (de trois sortes : P1, P2 et P3), portant notamment un pont roulant, et la couverture.

12- G. Oudotte, «Dépôts de locomotives à remises rectangulaires », Travaux, op. cit., p. 63-68. Ou encore : R. Bernis, «Les remises et ateliers du dépôt de locomotives de Châlons-sur-Marne », La Technique des travaux, janvier-février 1949, p. 9-16. Parmi les remises rectangulaires reconstruites, celle de Châlons-sur-Marne représente sans conteste l'ouvrage le plus intéressant, d'un point de vue constructif, et le plus spectaculaire, par ses dimensions. 
Les poteaux P1 constituent la façade intérieure donnant sur le pont tournant. Ils soutiennent l'élément de couverture correspondant et reçoivent les glissières des rideaux métalliques de fermeture. Les poteaux P2, situés à l'intérieur de la rotonde, supportent les efforts exercés par la couverture et reçoivent également l'appui du pont roulant. Les poteaux P3 de la paroi extérieure, en nombre triple des poteaux P1, sont de deux sortes. Les uns, qui se trouvent dans l'axe des voies, ne sont pas porteurs : ils peuvent être supprimés au cas où, pour des raisons d'exploitation, un nouvel accès à la rotonde serait ménagé ; les autres sont porteurs. Ce sont les fameux «V Laffaille», poteaux-coques à section en $V$ qui, grâce à la leur morphologie plissée, offrent une excellente solidité tout en exigeant peu de matières pour leur mise en œuvre. La couverture, quant à elle, est constituée d'un voile mince de béton armé de $7 \mathrm{~cm}$ d'épaisseur. Elle se compose, entre les poteaux P1 et P2, d'un voile conique plat, et, entre les poteaux P2 et P3, d'un voile parabolique raidi par des tirants. Enfin, l'imposante façade extérieure se compose d'éléments standards préfabriqués, en béton armé : trumeaux en $\mathrm{V}$ bien sûr, mais aussi allèges, châssis de vitres, linteaux et cornichesdéflecteurs.

\section{Une conception assistée par l'économétrie}

On l'a souligné, la modernité des rotondes réside en partie dans les nouvelles méthodes déployées pour leur conception. Outre leur configuration modulaire et normalisée, elles ont été l'occasion pour Laffaille d'expérimenter ses idées en matière d'économétrie appliquée à la conception architecturale ${ }^{13}:$ "Les rotondes, précise-t-il, ont été étudiées et réalisées en vue de déterminer les dimensions les plus économiques compatibles avec les programmes (dimensions des machines, ponts roulants intérieurs, gabarits, puissance d'éclairage naturel, encombrement du terrain, etc.). Tous ces facteurs ont été introduits dans des équations générales caractérisant le volume du bâtiment, et l'ensemble de ces fonctions, dans lesquelles nous avions introduit sous forme de paramètres les valeurs des salaires, des matériaux, des transports, etc., a conduit à une fonction globale dont l'étude mathématique a pu permettre la mise en évidence de solutions minima. C'est parmi ces solutions minima qu'on a choisi celle qui a donné les caractéristiques de ces bâtiments. Il y a eu là un très important travail mathématique, vingt fois

13- IFA, cote 36/2, lettre du 26 juillet 1948, de Laffaille à l'ingénieur Galland. Les calculs économétriques menés sont présentés dans une note de Laffaille de septembre 1947 (IFA 23/1) et, de manière très succincte, dans Le Génie civil, 1 ${ }^{\text {er }}$ mai 1948, p. 161164. 
plus important (en temps passé) que celui consacré habituellement à des calculs de résistance de matériaux. Cet effort était justifié puisqu'il s'agissait de bâtiments répétés en série et dont l'ensemble en France correspond à plus de deux cent mille mètres carrés. Si ce résultat a un « caractère somptuaire », cela prouve que des volumes peuvent créer dans l'espace une émotion artistique qui n'a pas de rapport avec un prix de revient. »

La recherche économétrique proprement dite a porté sur la clôture de la façade extérieure, de sa configuration et du nombre de ses éléments constitutifs, étant entendu que les données générales de dimension du projet découlaient du programme puisqu'elles étaient fonction, d'une part, des manipulations intérieures par pont roulant et, d'autre part, des conditions les meilleures pour l'évacuation des fumées par aspiration périphérique ${ }^{14}$.

\section{L'éclairage intérieur et l'évacuation des fumées}

La réponse technique et constructive apportée au problème essentiel de l'éclairage intérieur illustre parfaitement le caractère rationnel, voir scientifique, de la conception des rotondes. Ainsi, afin que les fumées des locomotives n'obscurcissent pas les surfaces vitrées, comme c'était le cas pour les anciennes remises rectangulaires éclairées par lanterneaux, les surfaces éclairantes ont été rejetées sur les façades extérieure et intérieure. L'intensité de l'éclairage naturel à l'intérieur du volume construit a été étudié scientifiquement en fonction des dimensions et de l'éloignement des surfaces éclairantes (fig. 5). L'étude a montré la nécessité de porter la hauteur des «V Laffaille » à $15 \mathrm{~m}$ et des poteaux P1 à $8 \mathrm{~m}$. Sur la paroi extérieure, la surface éclairante représente $75 \%$ de la surface totale de la façade.

Le nombre total de $\mathrm{V}$ périphériques et, donc, leur espacement, a été déterminé à la fois par l'étude de l'éclairage et par les calculs économétriques menés par Laffaille. De manière inattendue, les deux analyses ont abouti sensiblement au même résultat, à savoir un espacement proche de trois mètres entre chaque $\mathrm{V}$.

La collecte des fumées se fait à l'aide d'une hotte (fig. 6, 7, 8) placée directement au-dessus des cheminées des locomotives ainsi que, pour les fumées traînantes ou résiduelles, par des ouvertures ménagées à l'intrados des voûtes de couverture et qui donnent dans des gaines rayonnantes formant nervure. Ces fumées débouchent sur l'extérieur au niveau du sommet de la façade par l'intermédiaire d'un déflecteur

14- Précisions apportées par Laffaille dans sa note de 1947. 


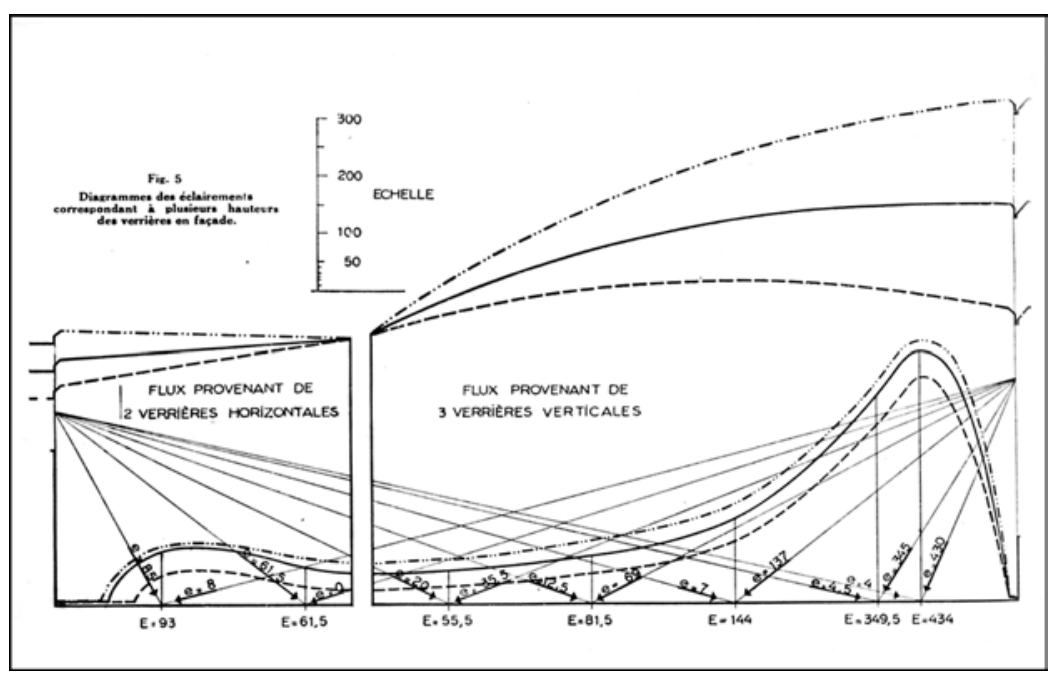

Figure 5. Essais sur l'intensité de l'éclairage naturel intérieur d'une rotonde en fonction de la hauteur de ses façades, 1945-1946. Coll. SNCF, Cl. N. Nogue.

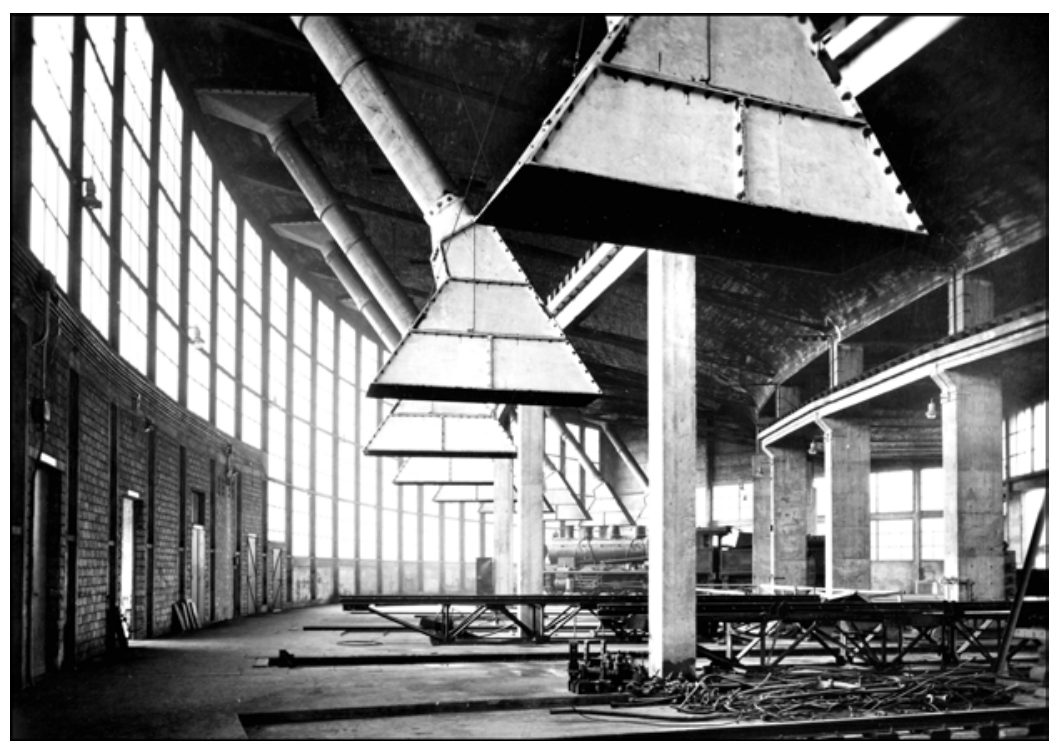

Figure 6. Vue intérieure de la rotonde de Poitiers avec ses hottes aspirantes en métal, ca 1948. Coll. et cl. SNCF. 


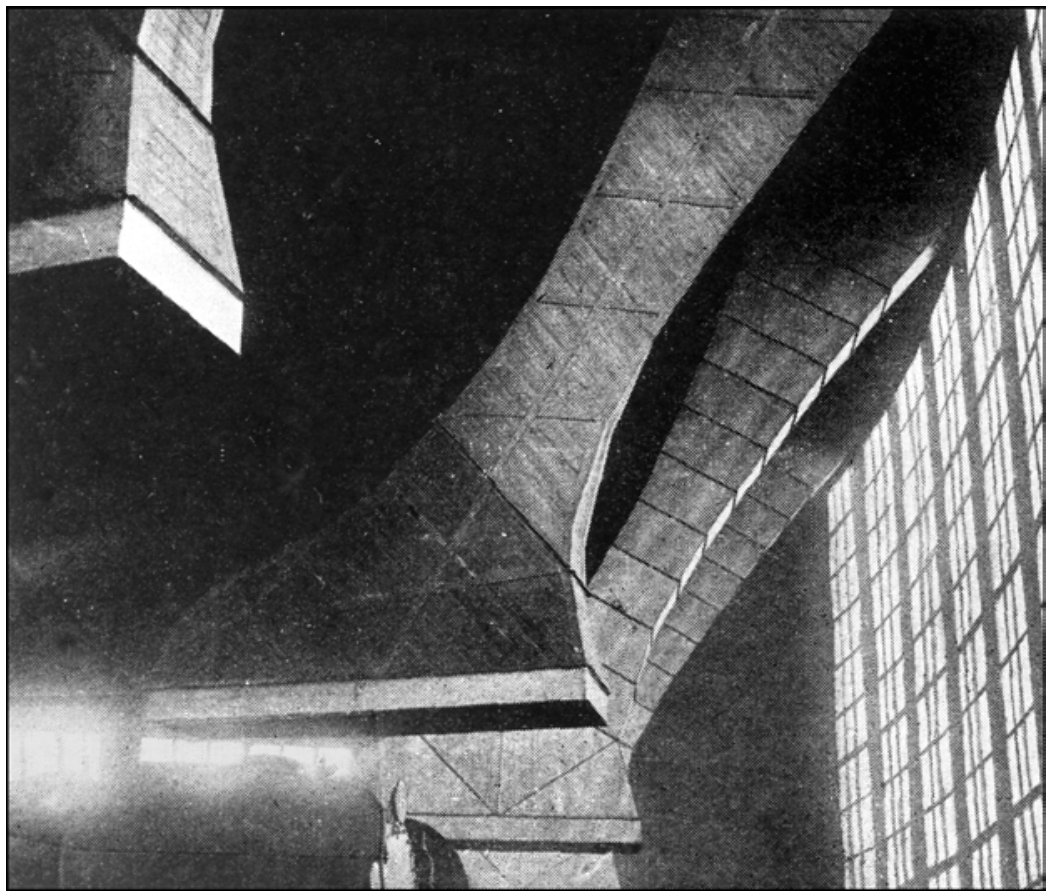

Figure 7. Vue de hottes aspirantes, vraisemblablement celles de la rotonde d'Avignon. Coll. et cl. SNCF.

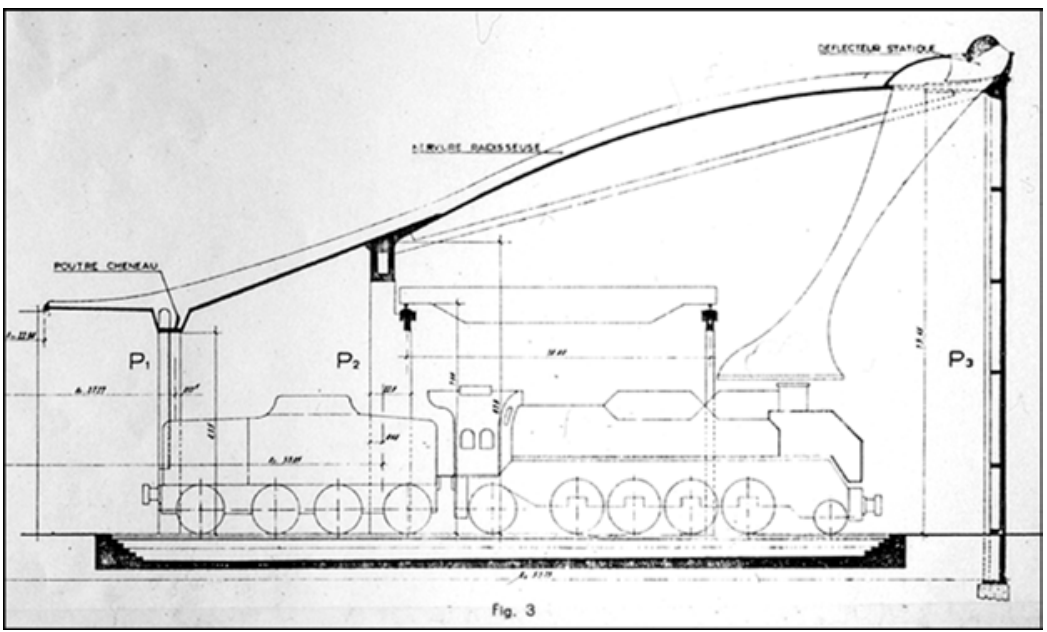

Figure 8. Coupe sur fosse de la rotonde d'Avignon avec la cheminée de la locomotive placée sous la hotte aspirante. Coll. SNCF, cl. N. Nogue. 


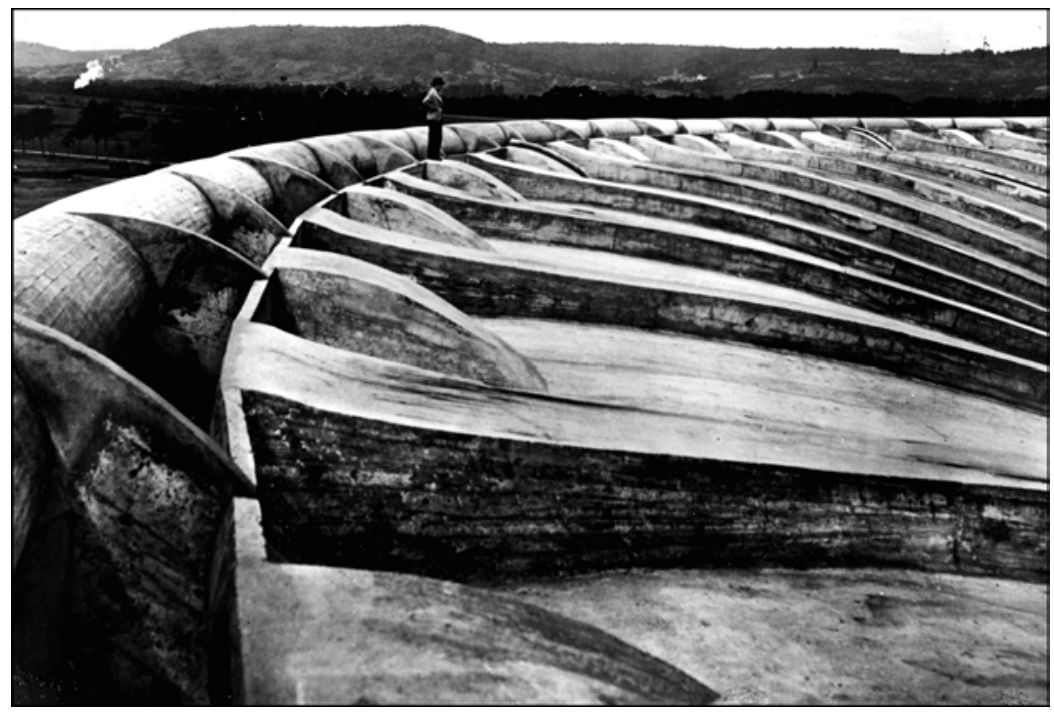

Figure 9. Détail de la toiture de la rotonde de Metz au niveau du " déflecteur statique " où débouchent, alternativement, les nervures creuses et les hottes aspirantes, 1948. Ces dispositifs, réalisés en béton brut de décoffrage, revêtent une plasticité saisissante. Coll. et cl. SNCF.

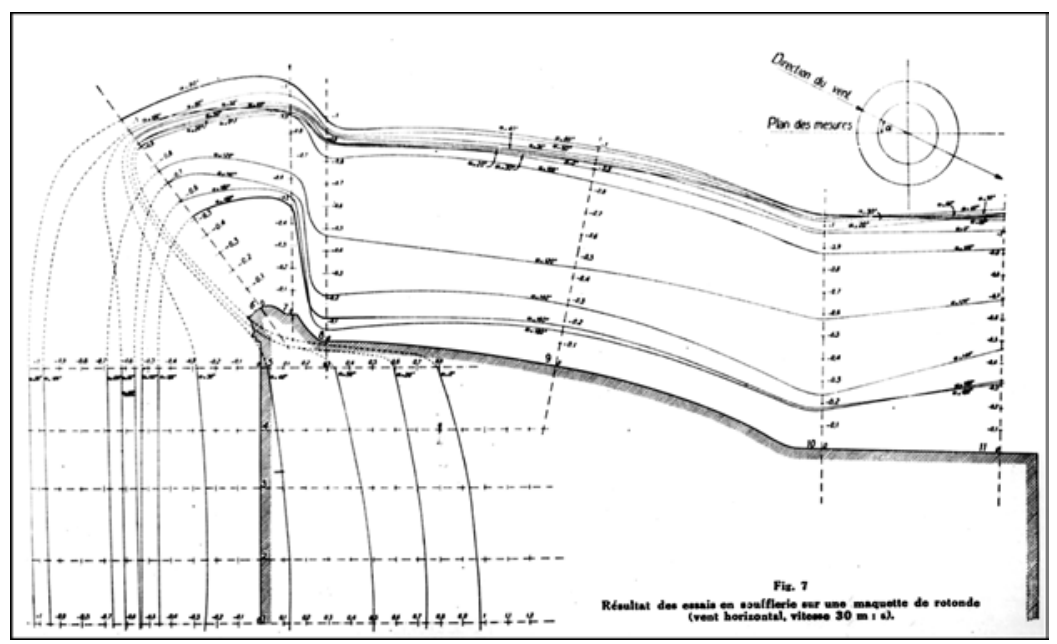

Figure 10. Essais en soufflerie pour tester l'efficacité du dispositif architectural d'aspiration naturelle de la fumée des locomotives grâce au "déflecteur statique " et au profil parabolique de la couverture, 1946. Coll. SNCF, cl. N. Nogue. 
statique (fig. 9) formant corniche et qui, par sa forme même et celle de la voûte parabolique, crée à cet endroit une zone permanente de dépression, accélérant l'évacuation des fumées. Ce dispositif original a été étudié et testé en soufflerie sur maquettes (fig. 10).

L'évacuation des fumées est facilitée, en outre, par l'élimination de la façade extérieure de toute pièce d'ossature horizontale qui aurait créé le long de la paroi des zones de remous, et par ailleurs, par l'accentuation des saillies verticales des $V$ qui permettent de conduire les filets d'air jusqu'à la zone supérieure de dépression.

Que ce soit au niveau de l'étude de l'éclairage ou à celui de l'évacuation des fumées, on voit que les nouvelles rotondes s'inscrivent dans un mouvement général contemporain de rationalisation de la conception architecturale et rejoignent, à ce titre, les études qu'ont pu mener, par exemple, Walter Gropius ou André Hermant sur l'éclairage naturel des logements, ou encore Gustave Lyon sur l'acoustique des salles de concert. Les recherches en soufflerie sur l'évacuation naturelle des fumées doivent aussi être rapprochée des préoccupations des modernes concernant l'architecture "bioclimatique », c'est-à-dire le contrôle du confort intérieur par des dispositifs architecturaux, à l'instar des brisesoleil de Le Corbusier ou de la ventilation naturelle de la «maison tropicale » (1949) de Prouvé.

À ce propos, la conception des rotondes repose entièrement sur une base technique et des déterminations d'ordre fonctionnel et économique. Leur «monumentalité » n'est pas le fait d'un architecte soucieux de symboliser ou magnifier leur fonction, comme c'est le cas par exemple de l'usine André-Blondel, par Théo Sardnal (un élève de Perret ${ }^{15}$ : cette dimension monumentale, involontaire pourrait-on dire, constitue la réponse logique apportée par l'ingénieur aux problèmes d'usage et de mise en œuvre posés par le programme ${ }^{16}$.

Il semble bien, toutefois, qu'un architecte de la division des Bâtiments ait participé à la conception de la rotonde-type. Il s'agit de Guy Brigaux, architecte D.E.T.P., dont le nom est mentionné par Robert Lévi dans un article présentant la rotonde d'Avignon ${ }^{17}$. Il est impossible de déterminer dans quelle mesure cet architecte est intervenu dans la conception des projets. En revanche, il est certain que l'ouvrage-type a fait l'objet d'un contrôle esthétique soigné. Robert Lévi précise à ce

15- Voir G. Monnier, op. cit., p. 383.

16- C'est aussi ce que précise Robert Lévi dans la Revue générale des chemins de fer, janvier 1948, $\mathrm{n}^{\circ}$ 1, p. 1-4 : "On confère ainsi à la construction, sans l'avoir cherché, un caractère monumental particulièrement remarquable. » 
sujet : «Des maquettes grandeur du profil des éléments portants, linteaux et corniches, furent établies et recommencées plusieurs fois jusqu'à ce que leur volume, l'éclairement de leurs surfaces, les ombres portées et les saillies accusant les lignes de construction constituent un ensemble harmonieux et à l'échelle humaine ${ }^{18}$. »

\section{Trois " ouvrages-types "}

La solution constructive mise au point a été déclinée, dans un premier temps, sous la forme de deux ouvrages-types qui diffèrent seulement par leurs dimensions : les rotondes type «P» (fig. 11) et « G». Les plans-types de la SNCF pour les deux variantes ont été élaborés en même temps : ils s'échelonnent de février à juillet 1945 pour le «type $\mathrm{P}$ », et de février à mai 1945 pour le «type $G{ }^{19}$. Durant l'année 1946, une « note de calcul-type » destinée aux entrepreneurs est élaborée pour la rotonde « $\mathrm{P}$ » par Laffaille en relation avec Vallette. Par ailleurs, les premiers résultats des essais en soufflerie effectués sur maquette sont établis le 14 décembre 194620. La rotonde type «P » correspond au plus grand nombre de rotondes réalisées (dépôts de Creil, Fives-Lille, Hirson, Laon, Longueau (rotonde 1), Mantes, Metz, Poitiers, Valenciennes et Villeneuve-Saint-Georges). Elle présente les principales caractéristiques suivantes :

- nombre maximum de voies rayonnantes (ou modules) : 48

- rayon intérieur : $36,50 \mathrm{~m}$

- rayon extérieur : $66,70 \mathrm{~m}$

- longueur des fosses : $25 \mathrm{~m}$

- hauteur de la façade, intérieur : $8 \mathrm{~m}$

- hauteur de la façade, extérieur : $15 \mathrm{~m}$.

La rotonde «type $G$ », quant à elle, présente des dimensions en plan plus importantes, afin d'accueillir des locomotives plus longues : son rayon extérieur atteint $71,70 \mathrm{~m}$, toutes les autres dimensions du type $P$ restant par ailleurs inchangées. La rotonde « type $G$ » abrite ainsi des fosses d'entretien de $30 \mathrm{~m}$ de long, contre $25 \mathrm{~m}$ pour le «type $\mathrm{P}$ ». Les rotondes de Béthune, Longueau (rotonde 2), et Somain ont été édifiées selon cette conception.

17- La Technique des travaux, $\mathrm{n}^{\circ}$ 11-12, novembre-décembre 1947, p. 337-346.

18- Ibidem. L'article est directement tiré d'un texte rédigé par Laffaille en septembre 1947 (voir dossier IFA cote 23/1).

19- Voir IFA, cote 22/1, pour la rotonde type P, et cote $25 / 7$, pour la rotonde type G. Il existe également des plans-types de l'IETP (cote 22/1), mais ils ne sont pas datés.

20- Voir IFA cote 23/1. D’autres essais furent menés en mars et mai 1947 (voir IFA cote 21/5). Peut-être étaient-ils destinés à tester la rotonde type «Pa » dont il est question au paragraphe suivant. 


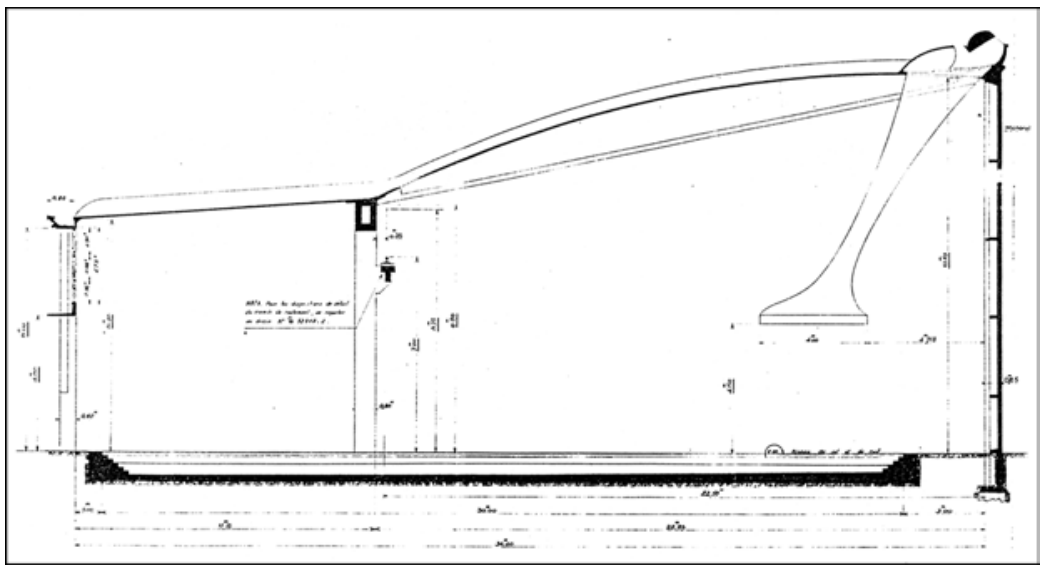

Figure 11. Coupe sur fosse $d^{\prime}$ une rotonde " type $P$ » avec la hotte d'aspiration des fumées reliée au "déflecteur statique ». Coll. SNCF, cl. N. Nogue.

Enfin, pour des raisons d'économie, une troisième variante a été élaborée, durant le second semestre $1947^{21}$ : la rotonde type «P abaissé » ou « $\mathrm{Pa} »$ (fig. 12 et fig. 13) qui reprend les dimensions en plan du type $\mathrm{P}$, mais réduit de deux mètres la hauteur de la voûte torique. La façade, de ce fait, s'élève à $13 \mathrm{~m}$ de hauteur contre $15 \mathrm{~m}$ pour le type $\mathrm{P}^{22}$. Comme la hauteur de la façade intérieure est maintenue inchangée, à $8 \mathrm{~m}$, la pente de la voûte conique plate se trouve de ce fait inversée : la voûte qui, pour les types P et G, s'élevait de un mètre entre la série des poteaux P1 et des piles P2, suit ici une pente descendante, le poteau P2 atteignant $7 \mathrm{~m}$ de hauteur. Seules deux rotondes ont été réalisées selon ce modèle, pour les dépôts de Culmont-Chalindrey et de Sarreguemines ${ }^{23}$.

21- Le directeur général de la SNCF approuva l'adoption du nouveau type de rotonde, lors d'une réunion tenue en mai 1947 (information recueillie dans une lettre de Robert Lévi au chef de la division des Voies et Travaux de la région Méditerranée, IFA cote 21/3)

22- Les plans-types définitifs de la rotonde type Pa sont datés de novembre et décembre 1947. Voir IFA, cotes $89 / 1$ et $89 / 2$.

23- Selon toute vraisemblance, les rotondes de Chalindrey et Sarreguemines sont les seules rotondes de type $\mathrm{Pa}$ car elles sont les dernières rotondes conçues. Nul doute que, s'il y avait eu d'autres rotondes élaborées à la suite de celles-ci, elles auraient suivi les configurations du type $\mathrm{Pa}$. 


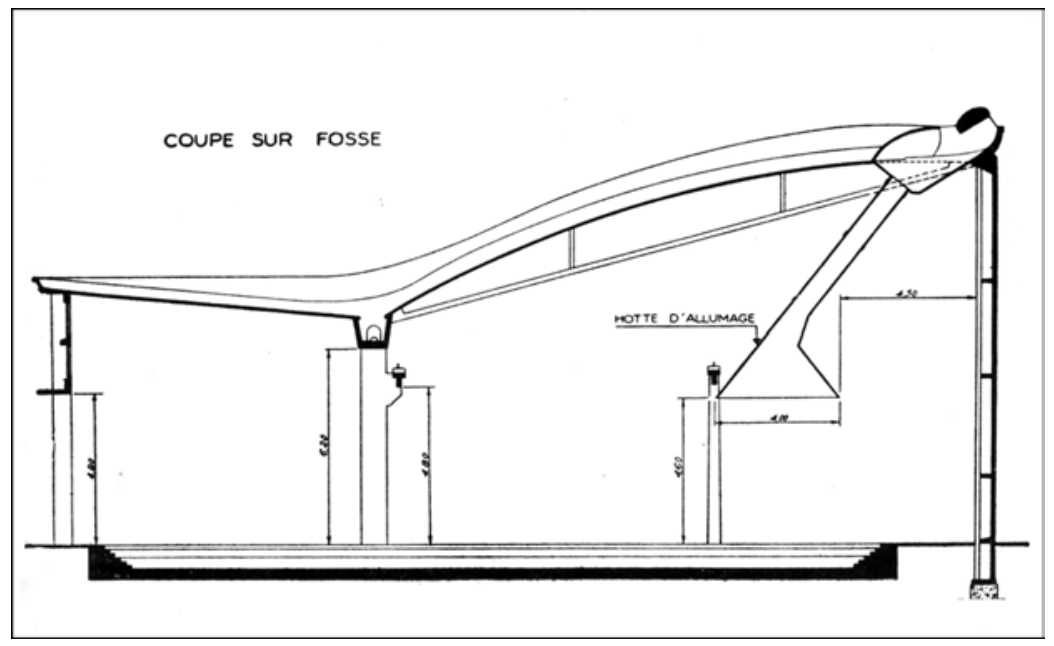

Figure 12. Coupe sur fosse d'une rotonde "type Pa». Coll. SNCF, Cl. N. Nogue.

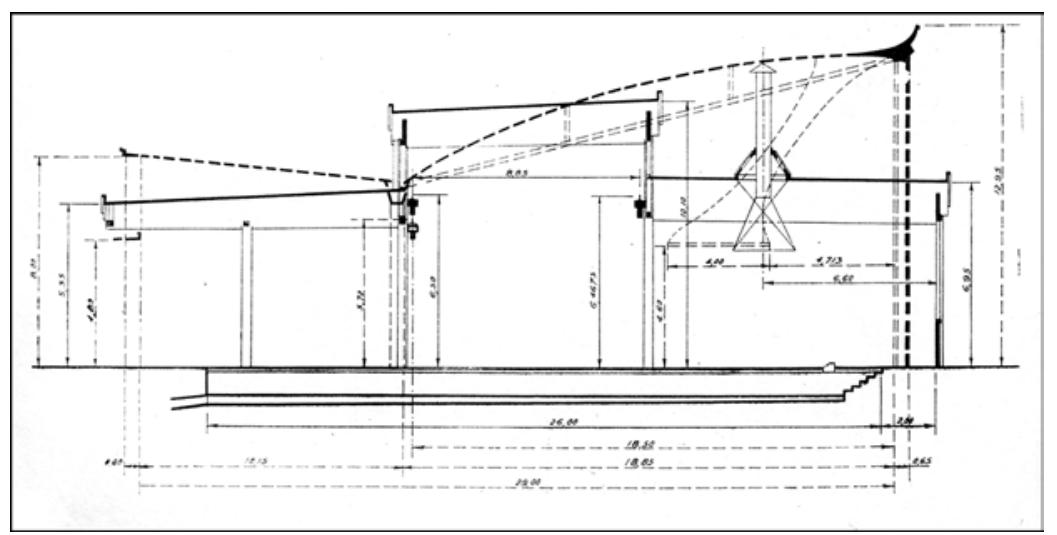

Figure 13. Coupes comparées d'une rotonde "PLM " des années 1920 et d'une rotonde "type $\mathrm{Pa}$ ", montrant le gain de volume intérieur du modèle de la Reconstruction sur celui d'avant-guerre et, par conséquent, les progrès obtenus pour les conditions de travail en termes de luminosité intérieure et de désenfumage. Coll. SNCF, cl. N. Nogue. 


\section{Deux projets avortés}

\section{Les "rotondes toriques"}

La genèse de la rotonde-type est, on l'a vu, mal connue : aucun document s'y rapportant ne subsiste dans les archives de l'ingénieur. On trouve la trace, cependant, d'un autre type de remise, non retenue par la SNCF, nommée "rotonde torique » (fig. 14), proche de la solution adoptée, au niveau de la structure et des éléments constructifs mis en œuvre, mais dont la principale caractéristique consiste en l'absence, à l'intérieur du volume couvert, de poteaux P2 : le voile de toiture se présente sous la forme d'un unique tore franchissant, sans appui intermédiaire, l'espace annulaire délimité par les trumeaux en $\mathrm{V}$ de la façade extérieure et par les poteaux P1 de la paroi intérieure ${ }^{24}$. En raison de sa configuration structurale, la rotonde torique ne peut accueillir de pont roulant. C'est vraisemblablement la raison pour laquelle cette solution n’a pas été retenue. Si sa façade extérieure s'élève à 13,50 m de hauteur, sa façade intérieure n'atteint que $4,80 \mathrm{~m}$. Deux versions ont également été déclinées : une rotonde de 27,50 $\mathrm{m}$ de portée (correspondant au type $\mathrm{P}$ ) et une autre de $34 \mathrm{~m}$ de portée (type $\mathrm{G}$ ).

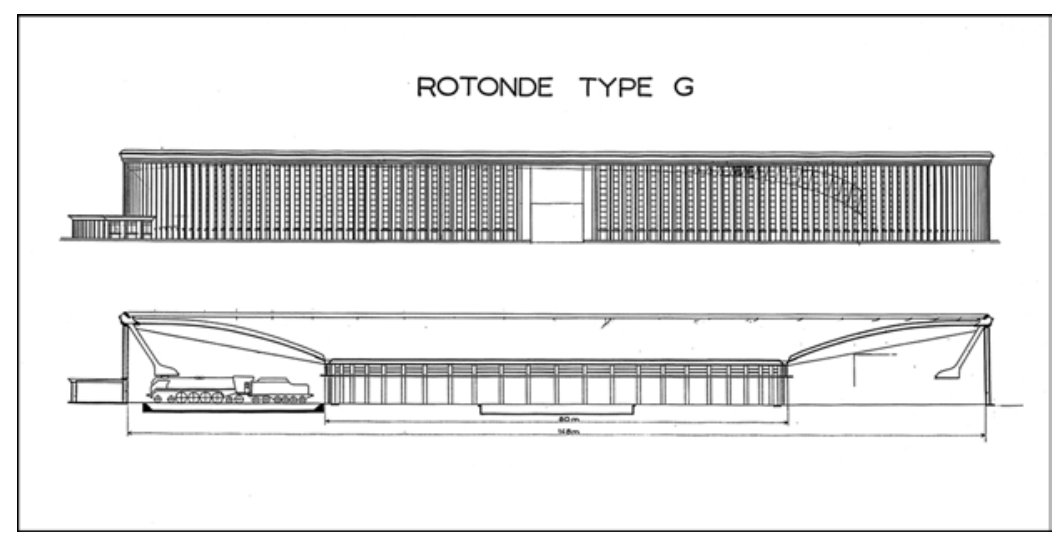

Figure 14. Rotonde type G dite "torique", prototype non retenu par les services de la SNCF. Coll. SNCF, cl. N. Nogue.

\section{Les " rotondes-bois"}

En 1946, Laffaille est également chargé de concevoir une rotonde couverte d'une charpente de bois, dont la réalisation est alors prévue sur le dépôt du Bourget ${ }^{25}$. L'ingénieur a proposé de conserver

24- Voir IFA, cotes $69 / 1$ et 208/5.

25- Voir IFA, cotes $80 / 2$ et $219 / 7$. 
les éléments d'ossature en béton armé des rotondes-types (poteaux P2, parois périphériques intérieure et extérieure) et d'adopter, pour la couverture, une voûte en bois collé et contreplaqué sous forme de coques, directement inspirée des coffrages mis au point par l'ingénieur-constructeur Molinié pour l'exécution de la rotonde de Metz ${ }^{26}$. Cependant, un autre projet a été étudié pour la SNCF, en 1947 : développé par l'entreprise A. Gorgeon, il prévoit de couvrir l'édifice annulaire d'une charpente courante, constituée de pannes, fermes et éléments de couverture en bois $^{27}$. En février 1948, la SNCF opte pour la solution de l'entreprise A. Gorgeon qui n'a toutefois pas été réalisée...

\section{Les exceptions à la règle de l'ouvrage-type}

\section{La rotonde d'Avignon}

La rotonde d'Avignon est la première rotonde réalisée. La SNCF entendait en faire « une application directe et complète du prototype $»^{28}$. La rotonde forme en effet un anneau complet et fut mise en œuvre, en un temps record, entre avril et décembre 1946, afin de mettre le prototype à l'épreuve du chantier et de pouvoir décider de sa généralisation. Mais, ironie du sort, l'exiguité du terrain a imposé de modifier légèrement les dimensions de l'ouvrage-type ainsi que sa structure, si bien que la première rotonde ne correspond pas exactement au projet-type, même si elle lui est totalement conforme du point de vue de ses dispositions générales et des procédés de construction.

Son diamètre s'est trouvé réduit à $108 \mathrm{~m}$, contre $134 \mathrm{~m}$ pour le modèle P. Mais la portée de la toiture, de 29 m, n'a pas été modifiée. En ce qui concerne la structure de la remise, on remarque, tout d'abord, le porte-à-faux de la couverture, ménagé par le retrait de 3,60 $\mathrm{m}$ à l'intérieur de l'édifice des poteaux P1 de la façade centrale. La voûte elle-même se distingue (fig. 15 et fig. 16). Elle est constituée d'un voile continu en forme libre, engendrée par la rotation d’une génératrice à double courbure : l'une convexe, l'autre concave. Cette couverture particulièrement harmonieuse n'accuse donc pas la rupture de celle de l'ouvrage-type qui, bipartite, comporte une portion conique plate accolée à une portion torique. Selon toute vraisemblance, la configuration de la voûte de la rotonde d'Avignon n'a pas été généralisée pour des

26- Voir lettre du 16 décembre 1946, de Laffaille à R. Gonon (IFA, cote 30/4).

27- Voir IFA, cotes $80 / 2$ et 219/7.

28- La citation ainsi que les informations figurant dans ce paragraphe proviennent de l'article de Robert Lévi publié dans La Technique des travaux, $\mathrm{n}^{\circ} 11-12$, novembredécembre 1947, p. 337-346. 


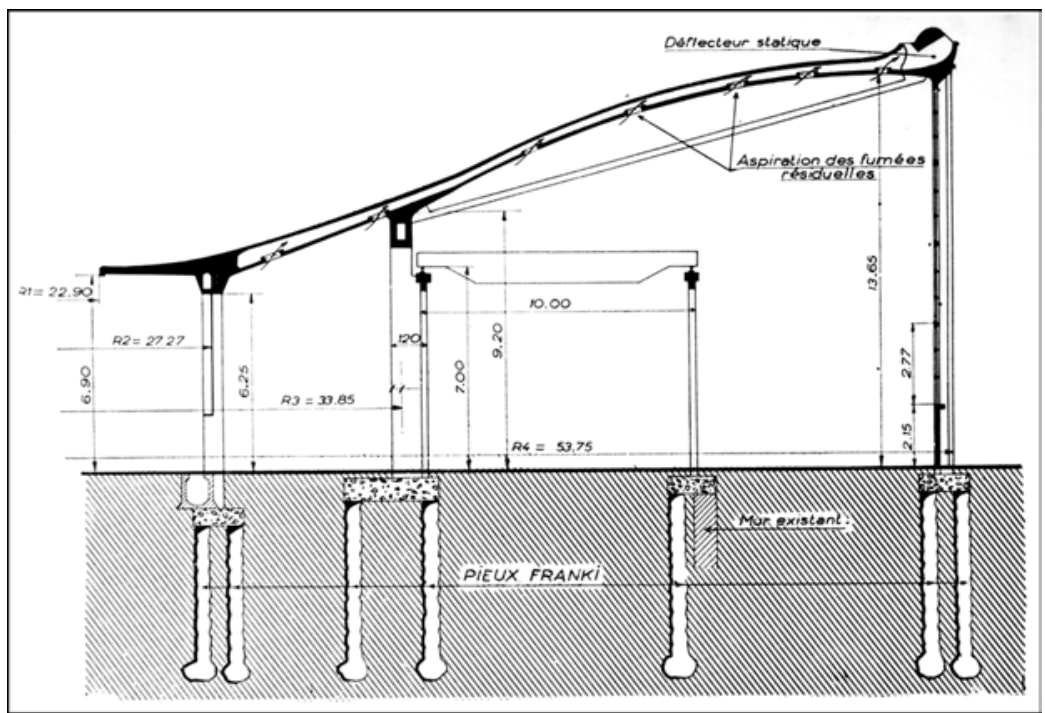

Figure 15. Coupe de la rotonde d'Avignon. Les gaines raidisseuses de la toiture sont creuses et, comme les hottes, débouchent sur le déflecteur statique de couronnement pour évacuer les fumées corrosives résiduelles. Coll. SNCF, cl. N. Nogue.

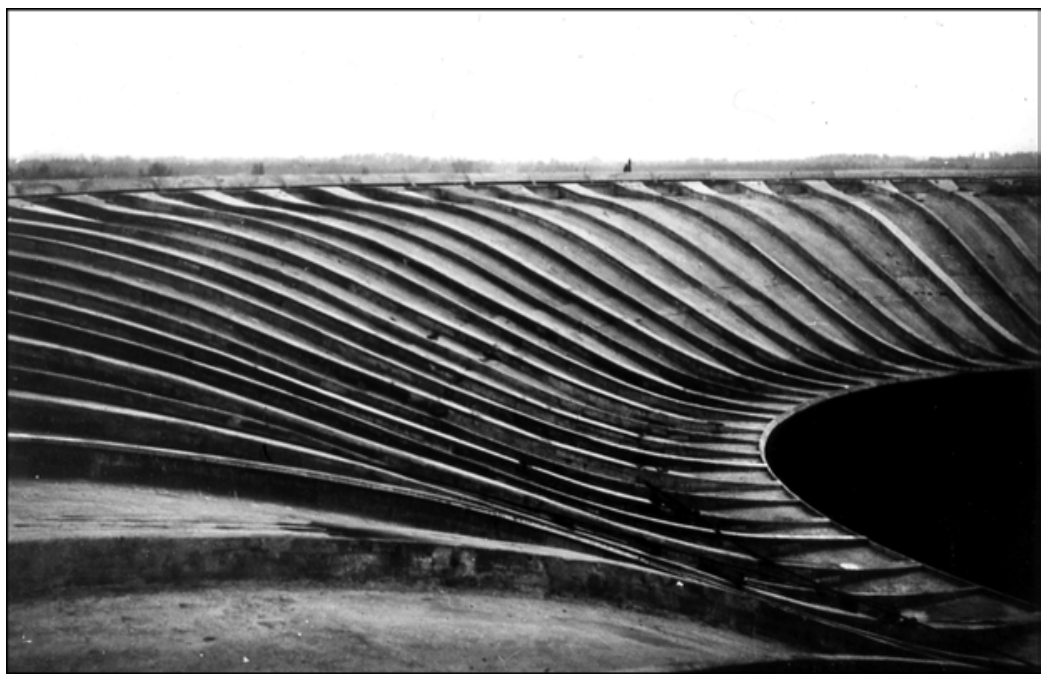

Figure 16. Détail de la couverture de la rotonde d'Avignon, épousant une forme libre, particulièrement élégante, qui n'est pas reprise pour les projets suivants. Coll. et Cl. SNCF. 
raisons d'économie et de facilité de calcul : présentant un porte-à-faux et une morphologie libre complexe, elle a été par la suite remplacée par une structure simplifiée. Mentionnons enfin que, à Avignon, la couverture n'est pas raidie par des tirants fixés entre les P2 et les trumeaux en $\mathrm{V}$, mais par des voiles pleins de béton armé épousant la courbe parabolique de la voûte.

\section{Deux rotondes "spéciales"}

Parmi les 19 remises réalisées, seules deux rotondes, celles de Cambrai et de Tourcoing, font réellement figure d'exceptions dans la mesure où elles ne correspondent pas à un des trois "modèles-type » élaborés. Toutes deux ont une structure semblable, sensiblement démarquée de celle des types $\mathrm{P}, \mathrm{Pa}$ et $\mathrm{G}$, et dont les dispositions ne furent pas établies en modèle-type, d'où leur appellation de « rotondes spéciales » (fig. 17). À Cambrai comme à Tourcoing, les poteaux P1 de la façade intérieure ont été supprimés : le voile de couverture se trouve donc en porte-à-faux sur l'espace central occupé par le pont tournant.

La conception initiale de Laffaille prévoyait, en 1946, que seule la poutre-gaine de la voûte assure la stabilité du porte-à-faux ${ }^{29}$. La réalisation cependant ne suivit pas, pour des raisons non éclaircies, cette solution élégante : le raidissement du porte-à-faux a été assuré par deux tirants extérieurs situés de part et d'autre de la gaine. Les tirants s'ancrent à l'extrémité du porte-à-faux et viennent se raccorder à la poutre-gaine de la voûte torique ${ }^{30}$.

Bien que les remises de Cambrai ( 9 voies) (fig. 18) et Tourcoing (21 voies) partagent le même schéma structural, leurs dimensions diffèrent sensiblement : à Cambrai, la voûte torique atteint une portée de $19,50 \mathrm{~m}$ et le porte-à-faux de $5,50 \mathrm{~m}$. Les façades intérieures et extérieures s'élèvent respectivement à $9 \mathrm{~m}$ et $15 \mathrm{~m}$. Avec une portée totale de $25 \mathrm{~m}$, cette rotonde représente la plus réduite des rotondes conçues par Laffaille. La largeur du secteur d'anneau de la rotonde de Tourcoing atteint, quant à lui, $30 \mathrm{~m}$. La portée du porte-à-faux est de $8 \mathrm{~m}$, celle du voile torique de $20 \mathrm{~m}$. L'édifice reprend, par ailleurs, les dimensions réduites du type « $\mathrm{Pa}$ », avec des façades intérieure et extérieure s'élevant respectivement à $7,50 \mathrm{~m}$ et $13 \mathrm{~m}$.

29- Voir, pour la rotonde de Cambrai, les dossiers cotes IFA 89/3, 220/2 et 220/3, et pour celle de Tourcoing, les dossiers cotes IFA 87, 88, 221/1, 221/2 et 221/6.

30- Laffaille décrit lui-même cette solution structurale dans une lettre, du 20 novembre 1946, adressée à Vallette (voir IFA, cote 6/4). 


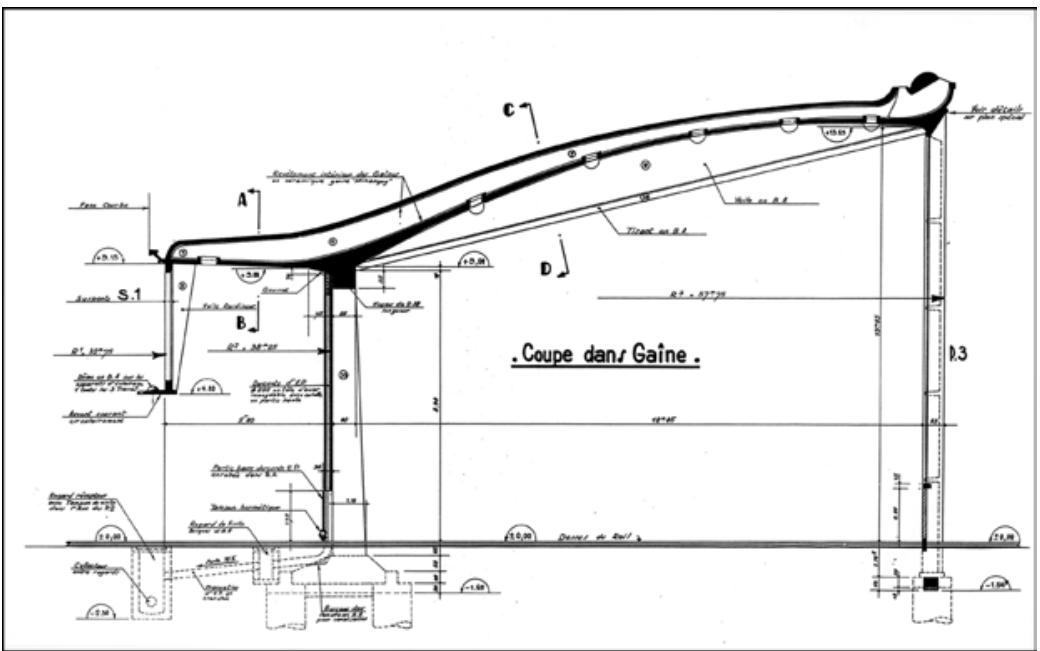

Figure 17. Coupe sur gaine d'une rotonde "spéciale». Coll. SNCF, cl. N. Nogue.

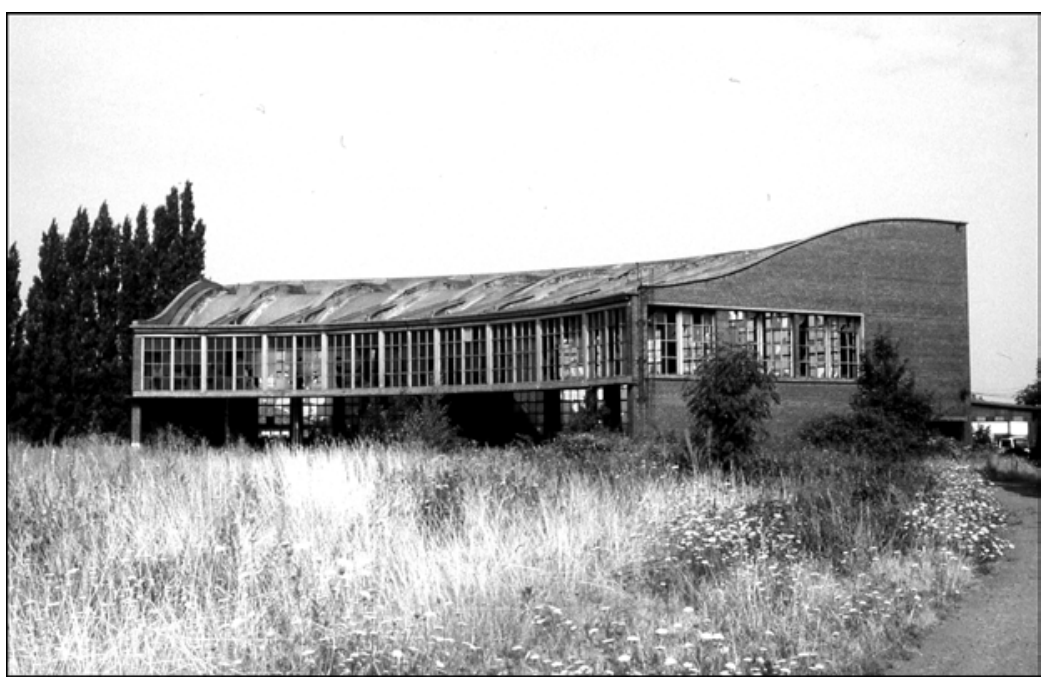

Figure 18. Vue de la rotonde "spéciale " de Cambrai, aujourd'hui détruite (cliché de 1994). Coll. et cl. N. Nogue. 


\section{La rotonde de Noisy-le-SeC}

Cette rotonde représente la dernière entorse à la règle des ouvrages-types : le besoin en remisage particulièrement important du dépôt de Noisy-le-Sec conduisit à adopter, pour cette remise, un type « $G$ modifié ». Le nombre de voies couvertes a été sensiblement accru par l'augmentation du diamètre extérieur, porté à $156 \mathrm{~m}$ contre $144 \mathrm{~m}$ pour le type $G$, la largeur de l'anneau restant inchangée $(34 \mathrm{~m})$. Complète, la rotonde de Noisy-le-Sec aurait abrité 56 voies (48 pour la rotonde type). Le projet initial prévoyait d'ailleurs l'édification d'un anneau complet, mais seulement 46 voies ont finalement été réalisées ${ }^{31}$. Cette remise n'en représentait pas moins la plus vaste des nouvelles rotondes, par les dimensions de son diamètre extérieur et par le nombre de voies couvertes.

\section{Un chantier d'ampleur nationale}

\section{L'organisation de la mise en œuvre ou le triangle opéra- tionnel : SNCF-Laffaille / IETP-entreprises}

L'édification de chaque rotonde a fait l'objet d'un appel d'offre spécifique afin de contenir au maximum les coûts de construction ${ }^{32}$. Laffaille avait alors en charge la vérification des dossiers d'exécution établis par les entreprises adjudicataires. La construction d'une rotonde établissait ainsi un réseau serré d'échanges d'informations entre les trois acteurs du projet : Laffaille (et son bureau d'études, l'IETP), l'entreprise adjudicataire et la SNCF (en particulier Vallette). C'est la SNCF, bien sûr, qui approuvait les dossiers d'exécution ; mais elle le faisait après le contrôle de Laffaille.

L'ingénieur-conseil décrit ainsi le circuit des dossiers d'exécution en vue de l'approbation par la $\mathrm{SNCF}^{33}$ : « Les entreprises envoient leurs dossiers à l'Arrondissement. De là, ils passent à la Région puis à la

31- M. Charton, agent SNCF au dépôt de Noisy-le-Sec, détient les archives de la rotonde que j’ai consultées le 5 juillet 1994.

32- Seule la rotonde de Metz ne fit pas l'objet d'un appel d'offres en règle, en raison des relations de confiance établies entre les agents SNCF locaux et l'entreprise Ballot à laquelle fut confiée la réalisation de l'édifice.

33- Cette description est rédigée par Laffaille dans une lettre du 31 août 1946 adressée à l'ingénieur-conseil de l'entreprise Delagneau, M. Millet, chargée de la construction de la rotonde de Hirson. L'entreprise n'ayant pas respecté la marche à suivre pour l'obtention de l'approbation des dossiers, Laffaille est amené à la décrire dans cette lettre (IFA, cote 5/1). 
division des Ouvrages d'art; M. Vallette me les communique et je les lui retourne avec mes observations. Pour gagner du temps, j’ai demandé aux entrepreneurs qu'en même temps qu'ils font leur envoi à l'Arrondissement, ils m'en remettent un double. Mais je ne donne en fin de compte mon avis que sur le dossier qui me parvient de chez M. Vallette. Tout ceci est fait pour permettre à l'Arrondissement de faire ses observations sur les conditions locales du travail, que nous ne pouvons connaître de Paris (implantations, égouts, fondations, etc ; et pour centraliser, à la division des Ouvrages d'art, tout l'ensemble des dossiers techniques. »

Pour certains projets, les relations entre les trois acteurs se trouvaient simplifiées dans la mesure où Laffaille était également choisi comme ingénieur-conseil de l'entreprise adjudicataire. Ce fut le cas notamment pour les rotondes de Creil, Fives-Lille, Mantes, Villeneuve-Saint-Georges, Metz et Tourcoing ${ }^{34}$. Pour les autres rotondes, les entreprises, soit possédaient leur propre bureau d'études (à l'instar des Établissements Limousin, adjudicataires de la rotonde de Laon, ou de l'entreprise Coignet, constructeur de la remise de Béthune), soit faisaient appel à un bureau d'études extérieur, comme la Société d'entreprises générales et de travaux publics qui, pour les études d'exécution de la rotonde de Poitiers, a fait appel au bureau d'études techniques Pelnard-Considère \& Caquot.

\section{Variations sur un thème}

Bien que toutes les rotondes adoptent les mêmes configurations générales, aucune n'est absolument identique du fait, en premier lieu, de l'existence de trois ouvrages-types différents, et des modifications qui leur ont été quelquefois apportées (rotondes d'Avignon, Cambrai, Noisyle-Sec et Tourcoing), mais surtout en raison de la flexibilité de la solution constructive qui s'adapte aux besoins locaux de remisage. On a ainsi des rotondes abritant, au minimum, neuf voies (rotonde de Cambrai), et au maximum 46 voies (rotonde de Noisy-le-Sec).

Dans certains cas, quelques retouches ont également été apportées aux plans-types. Ainsi, les rotondes d'Avignon, Longueau (rotonde 1) (fig. 19 et fig. 20) et Tourcoing présentent des pans de leur façade extérieure en retrait par rapport à la ligne circulaire des modèles-types. Ces remises, en effet, bordent la limite de l'emprise SNCF sur laquelle elles ont été édifiées. Leur pont-tournant central est situé de telle manière que leur façade extérieure, si elle suivait le modèle du plan-type, dépasserait sur

34- Voir IFA, cote 22/1, la liste des entreprises et de leur ingénieur-conseil. Cette liste, qui ne mentionne que 13 rotondes, n'est pas complète. 
certaines portions de leur périphérie les limites de leur terrain. Le problème a été résolu en opérant sur cette façade un, voire plusieurs, décrochements correspondant à la portion trop avancée de l'édifice. Les rotondes d'Avignon et de Longueau présentent un seul retrait de cette nature, tandis que deux sont à noter à Tourcoing.

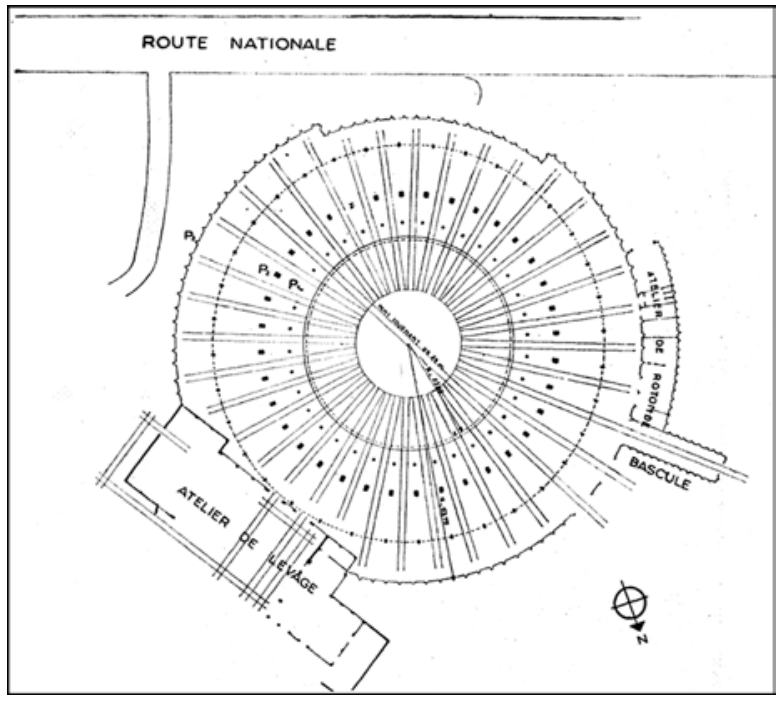

Figure 19. Plan de la rotonde d'Avignon avec sa façade en retrait. La rotonde est accolée à un atelier d'entretien également conçu par Laffaille. Coll. SNCF, cl. N. Nogue.

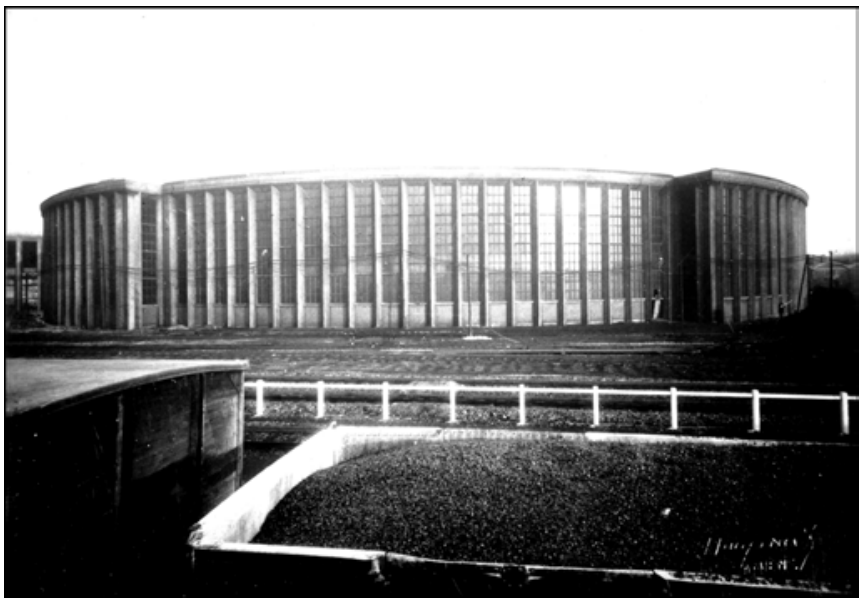

Figure 20. Rotonde de Longueau. Vue sur la façade de la partie en retrait, n.d. Coll. et cl. SNCF. 


\section{Les mises en œuvre}

L'exécution des dix-neuf rotondes, opérée par presqu'autant d'entreprises différentes, a donné lieu à des variantes dans la mesure où toute liberté était accordée aux constructeurs pour la définition des modes de mise en œuvre. Dans un article paru dans la revue Travaux ${ }^{35}$, $\mathrm{R}$. Gonon, ingénieur en chef de la région nord de la SNCF, relève les différentes façons dont les entreprises ont résolu la question de l'exécution des rotondes, dans sa région ${ }^{36}$. Il note en particulier qu'on n'a pas toujours recouru à la préfabrication des poteaux en $\mathrm{V}$ : «Certaines entreprises ont considéré qu'il était plus économique de réaliser les poteaux P3 par coulage sur place : c'est ainsi que, à Laon, l'entreprise Limousin s'est servi, pour couler les poteaux P3, de deux échafaudages roulants en forme de tour. Elle coulait ainsi six poteaux tous les dix jours. »

Quoi qu'il en soit, la mise en œuvre des rotondes (fig. 21, 22, 23, $24,25,26)$ s'est opérée, de manière générale, en deux phases, selon le schéma suivant ${ }^{37}$ :

- dans un premier temps, les poteaux P1 et P2 sont coulés en élévation. Simultanément, les poteaux P3 sont préfabriqués et démoulés au sol. Généralement, la portion conique de la couverture, soutenue par les poteaux P1 et P2, est également réalisée lors de cette première phase du chantier ;

- par la suite, le voile torique (de section parabolique) et la façade extérieure sont mis en œuvre. Pour l'exécution de la couverture, les entreprises ont réalisé des coffrages mobiles spectaculaires qui se déplaçaient sur des chemins de roulement circulaires, parallèles aux façades. $\mathrm{Au}$ moment où le coffrage roulant était mis en place et la portion torique coulée, on dressait le nombre de poteaux P3 correspondant au développement du coffrage. Étaient alors ajustés les autres éléments préfabriqués de la façade : linteaux, allèges, châssis des vitres et éléments du déflecteur statique formant corniche.

Initialement, Laffaille ne devait pas intervenir directement au niveau de la mise en œuvre des rotondes, c'est-à-dire sur le chantier, puisque sa responsabilité se limitait à celle d'un ingénieur-conseil. On a vu cependant qu'il exerçait un certain contrôle sur les entreprises dans le

35- Travaux, $\mathrm{n}^{\circ} 167$ bis, op. cit., p. 57-62.

36- Ibid., p. 61.

37- On reprend ici la description de la mise en œuvre - exemplaire - de la rotonde d'Avignon que fait M. Brigaux dans Le Bâtiment à la SNCF, $\mathbf{n}^{\circ} 10,1948$, p. 36-46. 


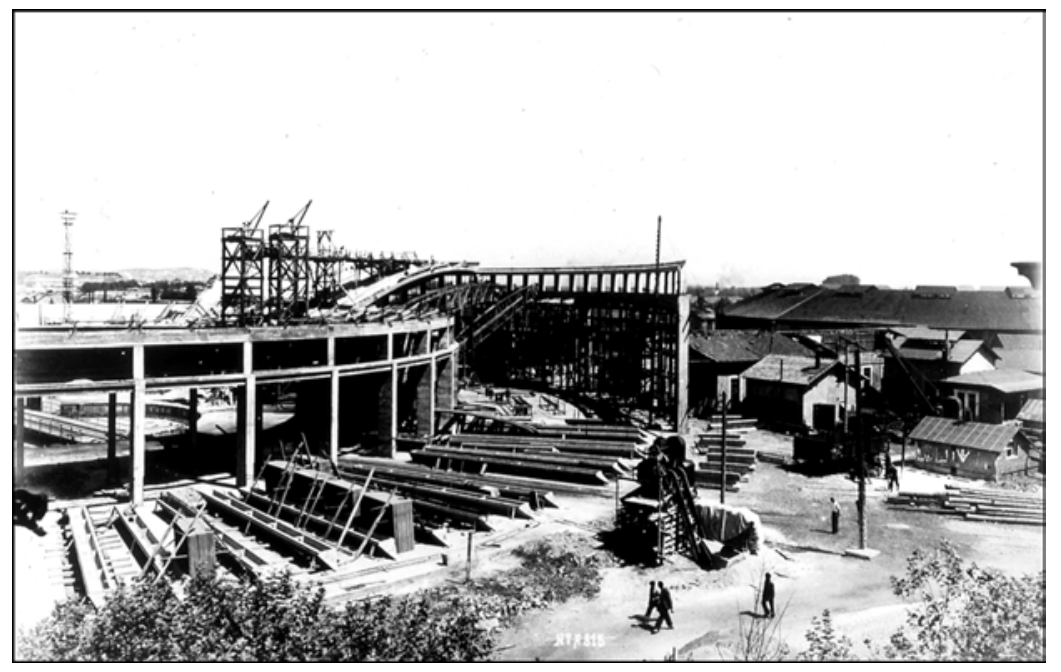

Figure 21. Chantier de la rotonde d'Avignon, 1945-1946. Les poteaux centraux $\mathrm{P} 1$ et $\mathrm{P} 2$ ont été coulés en place. On remarque à terre les "V Laffaille" préfabriqués en attente de levage alors que la couverture est en cours de mise en oeuvre. Coll. et Cl. SNCF.
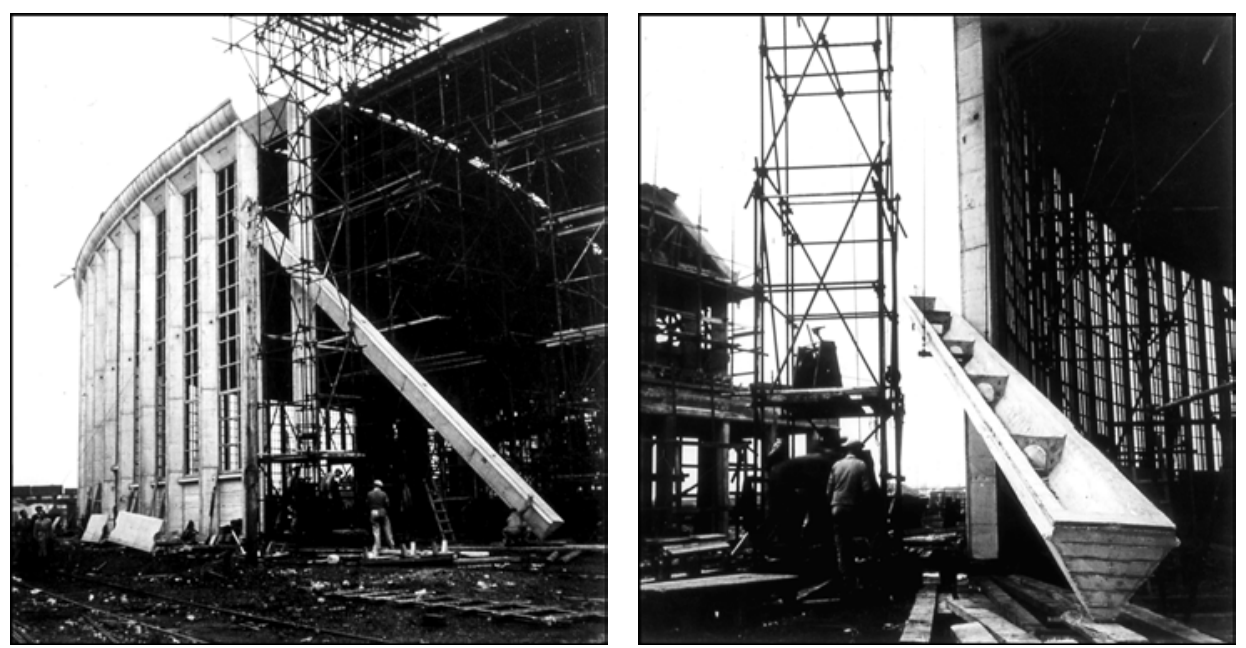

Figures 22 et 23 . Levage d'un $V$ de façade préfabriqué, 1946. Coll. et Cl. SNCF. 

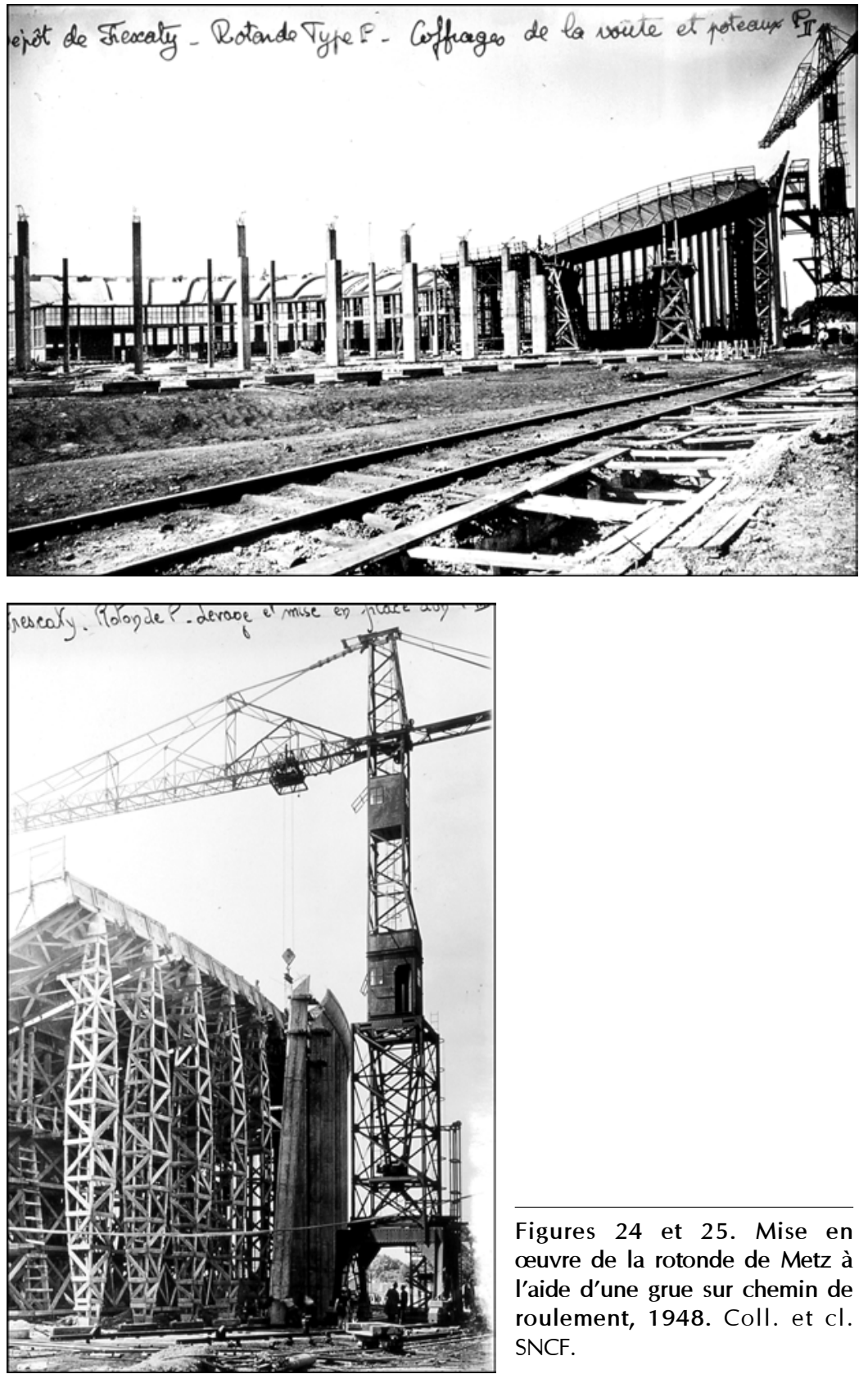

Figures 24 et 25. Mise en œuvre de la rotonde de Metz à l'aide d'une grue sur chemin de roulement, 1948. Coll. et $\mathrm{cl}$. SNCF. 


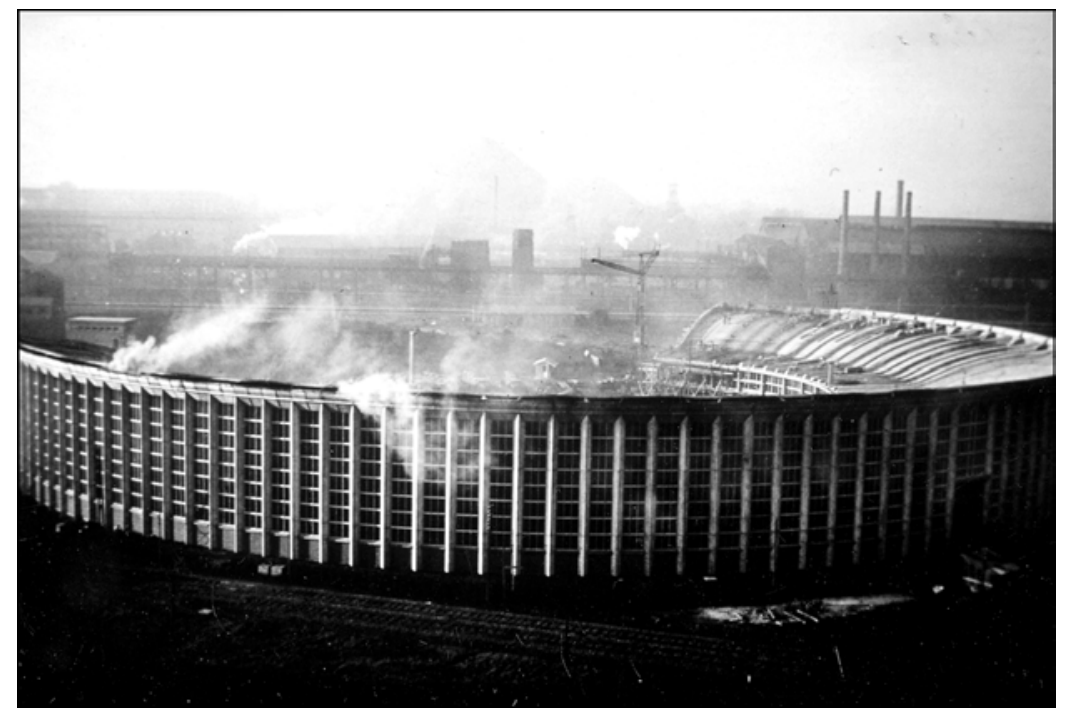

Figure 26. Fin de la mise en œeuvre de la rotonde de Valenciennes, exploitée avant son achèvement, ca 1948. Coll. et cl. SNCF.

cadre de la vérification de leurs dossiers d'exécution. Toutefois, à la suite d'un accident grave survenu sur le chantier de la rotonde de Creil, le 24 avril 1947, Laffaille fut amené à opérer un contrôle plus serré de la mise en œuvre : il envoya à toutes les entreprises une " note générale » reprenant les conditions à respecter pour l'exécution des rotondes, et s'est rendu, vraisemblablement, sur les différents chantiers afin de vérifier leur bonne conduite ${ }^{38}$. L'ingénieur eut également en charge de contrôler « les études, notes de calculs et plans des cintres, échafaudages et apparaux de levage, relatifs à l'exécution des rotondes $»^{39}$.

\section{Un chantier de sept ans}

(Fig. 27, 28, 29, 30, 31, 32, 33)

La première rotonde édifiée, celle d'Avignon, a aussi été celle dont la mise en œuvre fut la plus rapide : neuf mois, d'avril à décembre 1946. À l'opposé, l'exécution de la rotonde de Lille, a duré environ cinq ans, de 1947 à 1952 ! L'année 1952 marque aussi la fin du programme de construction des rotondes qui s'étendit, donc, sur sept ans.

38- C'est en tout cas ce qu'il laisse entendre dans une lettre, datée du 28 avril 1947, adressée à l'ingénieur Gonon (IFA, cote 7/2).

39- Voir lettre du 18 sept. 1947 de Laffaille à l'entreprise Labalette (IFA, cote 14/3). 
La durée exceptionnelle du chantier de Lille s'explique par le fait que, à l'instar des remises de Creil, Noisy-le-Sec et Tourcoing, la rotonde lilloise a été exécutée en plusieurs phases ${ }^{40}$. La première phase (1947-1948) a consisté en l'exécution d'un secteur de six voies au NordOuest du projet final, puis, en 1948-1950, d'une portion distincte de quatorze voies au Nord-Est. Enfin, en 1951-1952, un secteur de vingt voies a été bâti qui unissait les deux premières portions séparées. Les deux premières phases ont été réalisées par l'entreprise Pérignon, la troisième par l'entreprise Grands travaux de France et d'Outre-mer. Mais il s'agit là, bien sûr, d'une exception. En fait, la grande majorité des rotondes a été édifiée en une seule phase, par une seule entreprise, entre 1947 et 1948.

\section{Un programme amputé}

Les dix-neuf rotondes exécutées faisaient partie d'un programme de réalisation qui s'est en fait trouvé sensiblement amputé. Bon nombre de plans de dépôts, correspondant aux projets initiaux de la SNCF, indiquent la présence d'une autre rotonde adjacente, qui n'a pas été réalisée, ou un nombre de voies couvertes supérieur ${ }^{41}$. Ainsi, le projet d'origine de la remise de Noisy-le-Sec comportait cinquante-six voies (c'est-à-dire une rotonde complète, annulaire), mais quarante-six furent exécutées. De même, on prévoyait une rotonde de quarante-quatre modules à Somain et seuls trente-six ont été construits. À Chalindrey, sur les quarante-six voies initiales, trente-sept furent réalisées. Par ailleurs, les premiers projets pour les dépôts de Creil, Lille, et Valenciennes prévoyaient l'implantation de deux rotondes adjacentes, une de type $G$ et l'autre de type P. Là encore, le projet a été modifié et recadré sur la construction d'une seule rotonde, de type P. On note également la suppression pure et simple de certains projets, notamment celui de la rotonde de Carnoules (Var) dont l'étude fut avancée, au-delà du choix de l'entreprise, jusqu'au dossier d'exécution ${ }^{42}$. D'autres remises étaient également projetées à Belfort et à Toulon ${ }^{43}$.

40- En général, la mise en œuvre s'effectuait en plusieurs phases (deux dans les autres cas que la rotonde de Lille) pour des raisons économiques : lorsqu'elle existait encore, l'ancienne rotonde était conservée, et l'on ne construisait qu'une portion du projet, puis, dans un second temps, l'ancienne rotonde était rasée, et la construction de la nouvelle remise était alors reprise

41- J'ai en effet pu consulter les archives de chaque région de la SNCF, entre 1994 et 1995. 42- Voir IFA, cotes $6 / 3$ et $15 / 3$.

43-La rotonde de Belfort devait être de type «P abaissé » (voir lettre de Laffaille à Peirani du 6 août 1948, IFA cote 23/1). La rotonde de Toulon est mentionnée sur une liste de références de Laffaille, datée de novembre 1947. Ces deux rotondes ne firent l'objet, semble-t-il, d'aucune étude. 


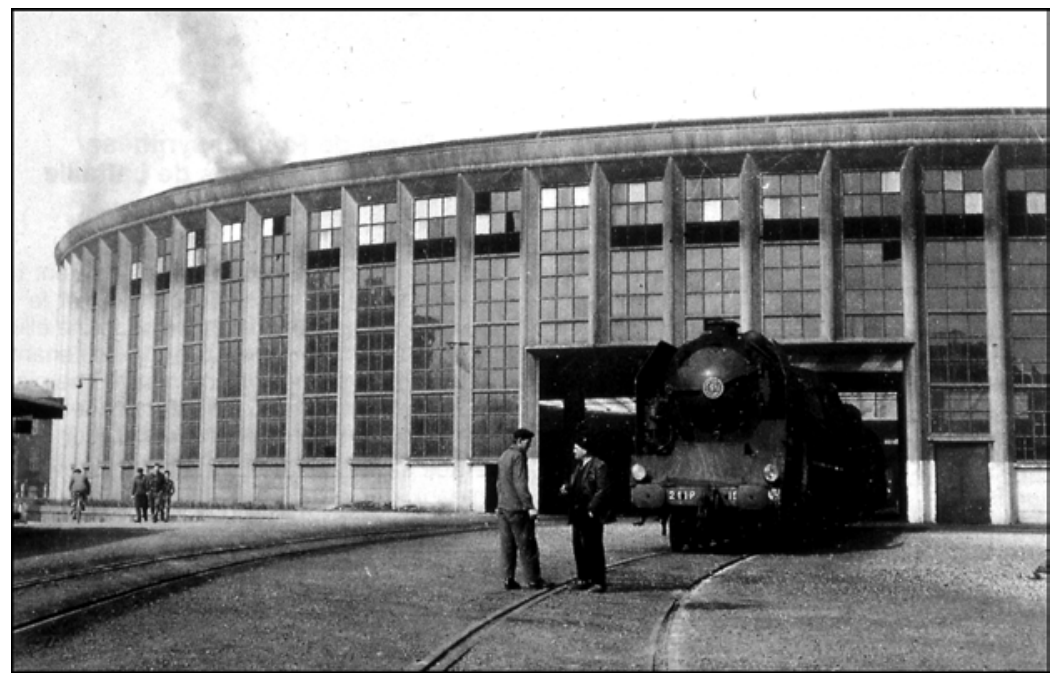

Figure 27. Façade de la rotonde d'Avignon, ca 1947. Cl. La Vie du Rail/Robert Long.

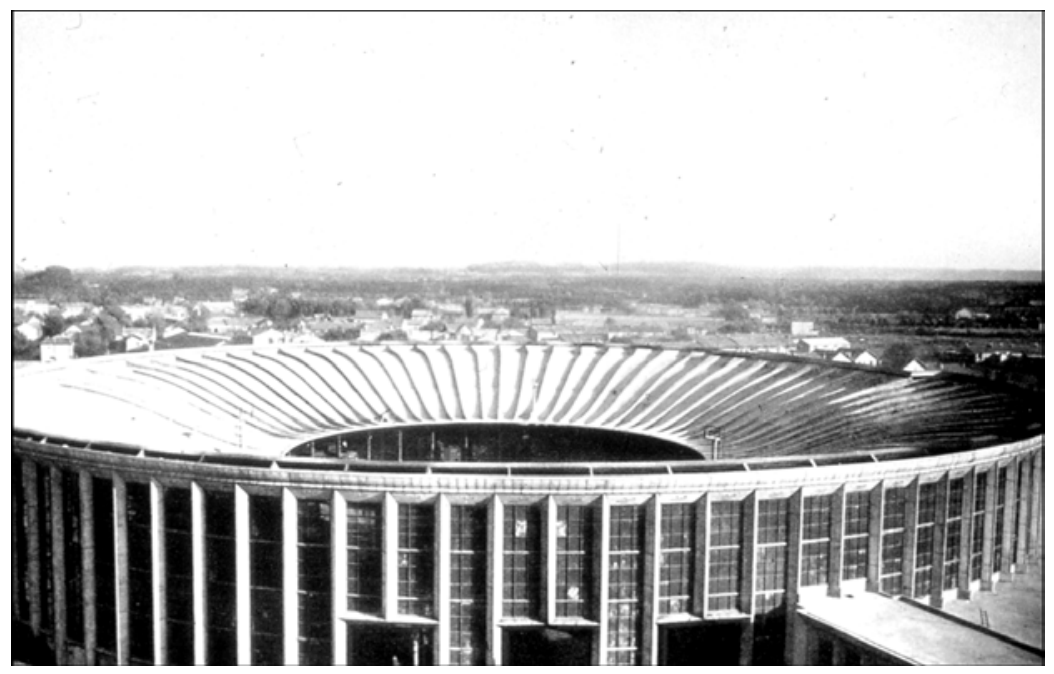

Figure 28. Vue générale de la toiture de la rotonde d'Avignon. Coll. et $\mathrm{cl}$. SNCF. 


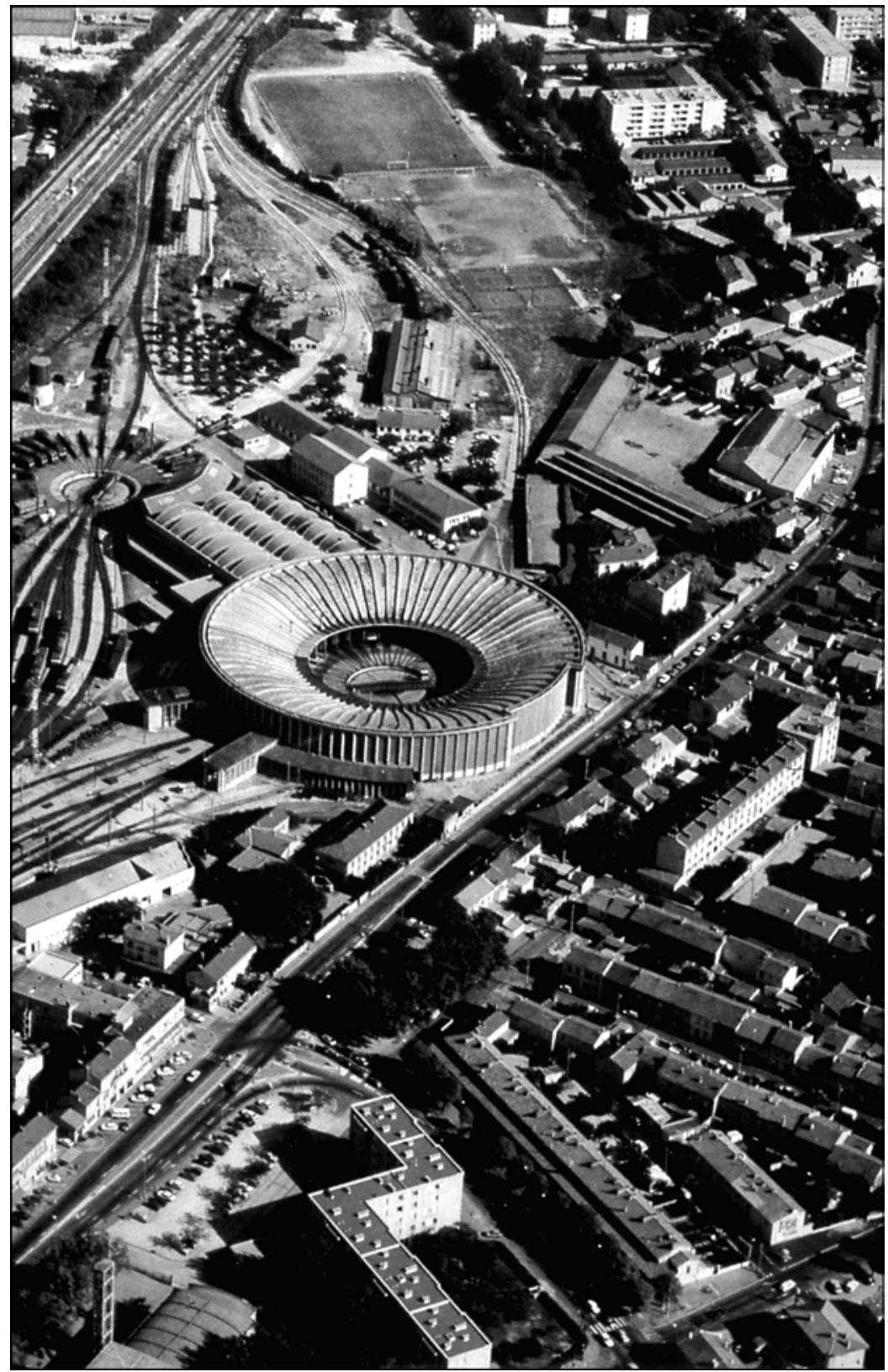

Figure 29. Vue aérienne de la rotonde d'Avignon dans le tissu urbain. Coll. et cl. SNCF. 


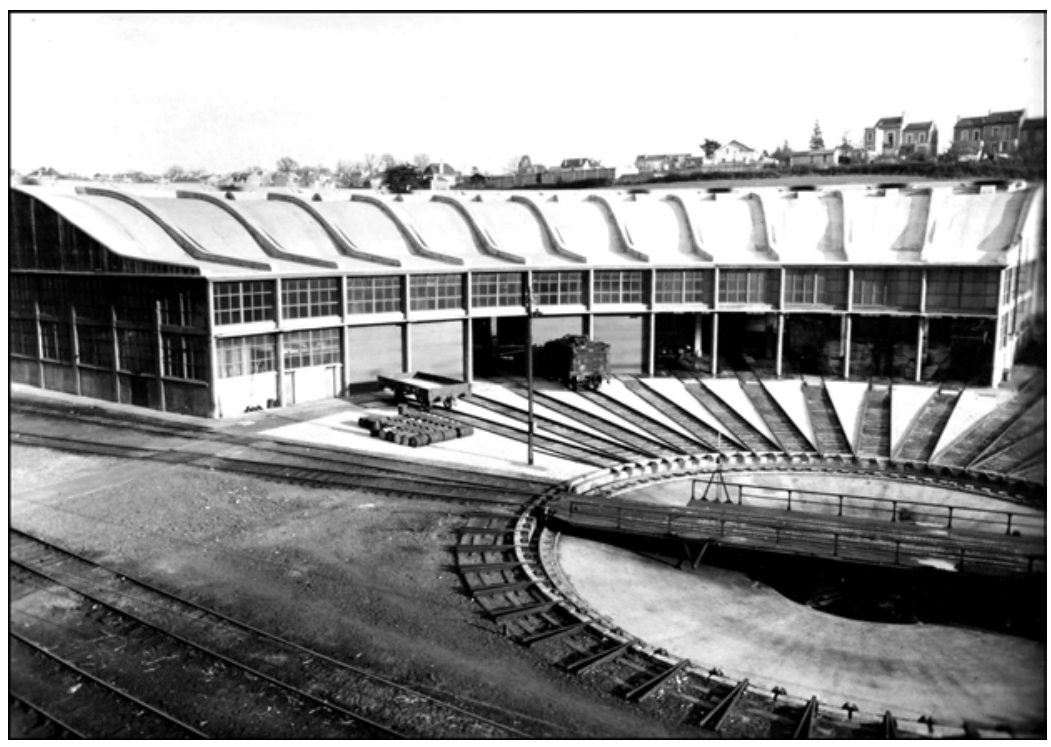

Figure 30. Vue de la rotonde de Poitiers avec son pont tournant, ca 1948. Coll. et cl. SNCF.

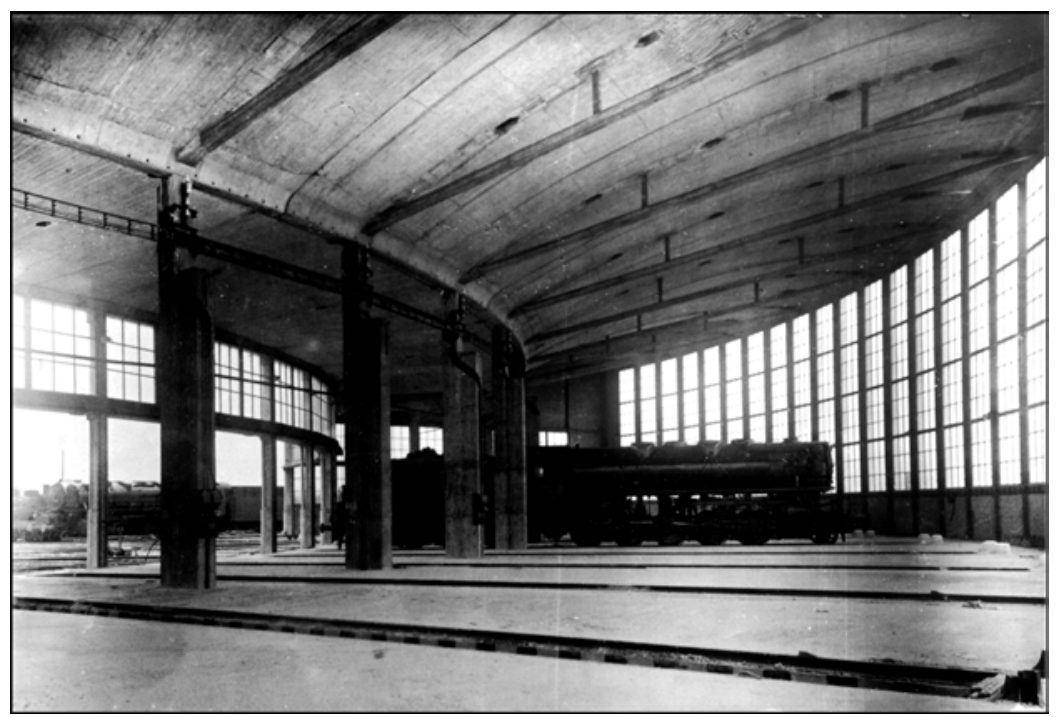

Figure 31. Vue intérieure de la rotonde Metz, 1948. Coll. et cl. SNCF. 


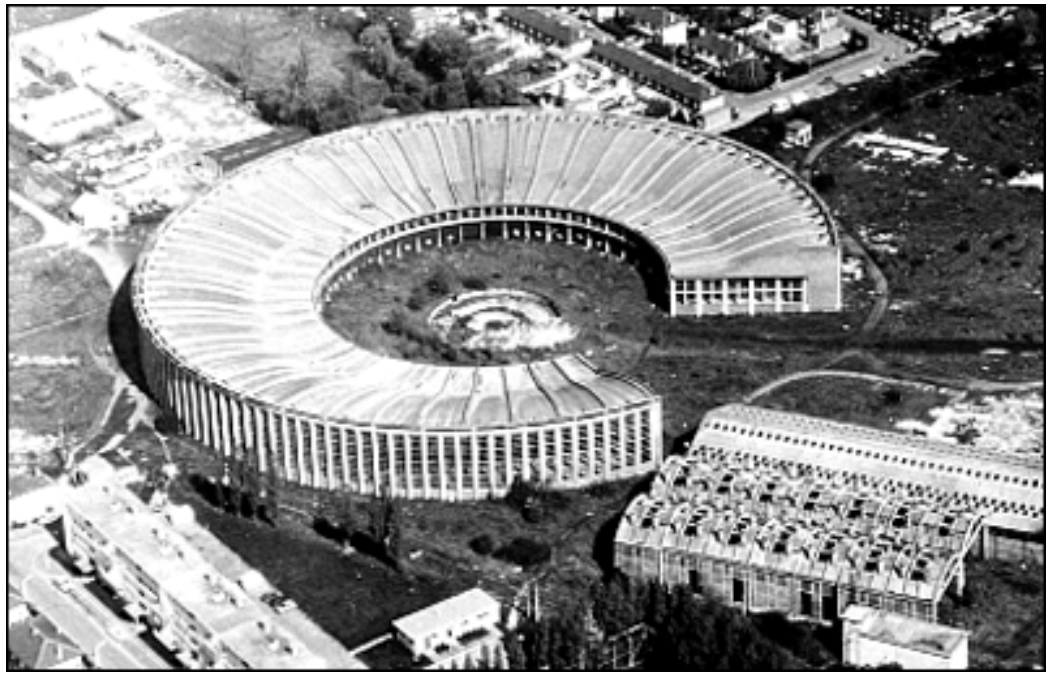

Figure 32. Vue aérienne de la rotonde de Béthune désaffectée dont le pont tournant a été démantelé, n.d. Coll. et cl. SNCF.

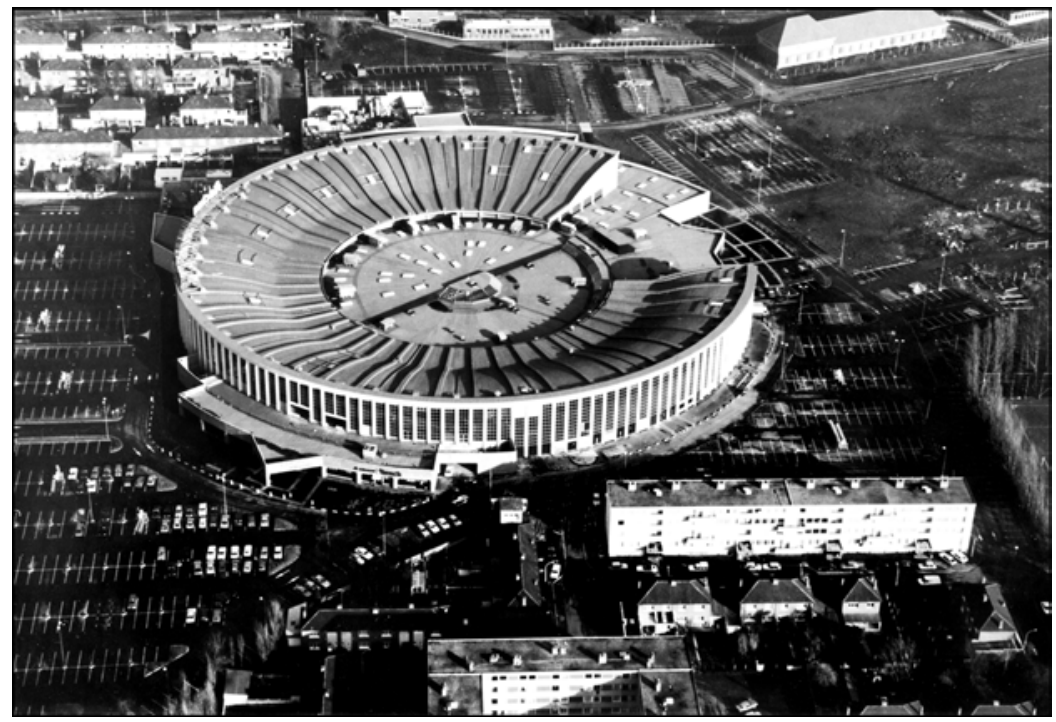

Figure 33. Vue aérienne de la rotonde de Béthune aujourd'hui réhabilitée en centre commercial. Coll. et cl. SNCF. 
Aucun document ne mentionne les raisons de la réduction du programme initial de construction. Selon toute vraisemblance, elle fut décidée pour des raisons d'économie. L'adoption des rotondes de type «P abaissé », en mai 1947, s'inscrivait déjà dans cette logique. La compagnie nationale, il est vrai, avait tout intérêt à réduire au maximum l'importance d'un programme dont l'utilité était condamnée, à terme, par l'électrification en cours du réseau et les nouvelles motrices qui ne nécessitaient plus d'être remisées comme les locomotives à vapeur.

\section{Bilan d'une opération exemplaire}

Au vu des ambitions initiales de la SNCF (objectifs fonctionnels et économiques) et de l'ampleur du programme réalisé, un bilan général de l'opération s'impose, qui s'avère, globalement, très positif. D’un point de vue fonctionnel d'abord : les «performances » des rotondes conçues par Laffaille améliorent sensiblement celles des modèles plus anciens $^{44}$, non seulement en ce qui concerne l'éclairage naturel à l'intérieur de la remise, mais aussi en matière d'évacuation des fumées. Le dispositif original du «déflecteur statique » associé à celui des hottes aspirantes surclasse les dispositifs antérieurs.

Par ailleurs, l'objectif initial de concilier l'adoption de plans-types et les exigences locales d'exploitation a été atteint, ce qui constitue une preuve de la flexibilité des nouveaux "modèles unifiés » : onze rotondes sur dix-neuf sont de type $\mathrm{P}$, soit les deux tiers, quatre remises furent réalisées selon le type $G$, et deux d'entre elles, les dernières rotondes conçues, celles de Chalindrey et de Sarreguemines, suivent le type « $\mathrm{Pa} »$. Ainsi, seize rotondes sur dix-neuf, soit plus des trois quarts, constituent l'application stricte d'un modèle-type. Deux rotondes «spéciales », on l'a vu, présentent une configuration plus ou moins modifiée par rapport aux ouvrages-types. Leur conception, toutefois, dérive totalement de celle des prototypes.

L'adoption des plans-types a permis de réduire les frais d'études et de contrôler au plus près les coûts d'exécution. La spécialisation des équipes sur le chantier, la mécanisation de la mise en œuvre ainsi que l'utilisation d'éléments standards préfabriqués ont également fortement contribué à diminuer le prix de revient des remises. Bernard Laffaille précise à ce sujet ${ }^{45}:$ « Ainsi, a-t-on éliminé complètement sur les chantiers

44- Voir: Revue générale des chemins de fer, $\mathrm{n}^{\circ} 1$, janvier 1948, p. 1-4.

45- Voir le document, non daté, intitulé « Note sur l'intérêt des traçages précis » (IFA, cote 28/1), dans lequel l'ingénieur détaille les raisons pour lesquelles la mise en œuvre des édifices qu'il conçut pour le compte de la SNCF est économique. 
SNCF dont je m'occupe, ces opérations très onéreuses de boisage et de coffrage, dans lesquelles on assemble clou par clou les planches entre elles ${ }^{46}$. On moule au sol dans des coffrages métalliques des pièces pesant jusqu'à six tonnes, et on les met au levage en une demi-heure avec quatre hommes, alors qu'auparavant, pour exécuter un travail similaire, il fallait deux journées à quatre hommes, pour effectuer les boisages et les temps de coulée en plus. Les économies d'emploi de main-d'œuvre et de prix sont appréciables puisque sur les rotondes on a pu économiser plus de mille francs par mètre carré avec les types nouveaux - en comparaison des types anciens en béton armé, dont on a exécuté également quelques constructions en même temps. Cette économie représente plus de cent millions de francs à l'heure actuelle sur les différents chantiers. »

Dans le même esprit, la comparaison entre la façade extérieure des rotondes et celle des bâtiments en béton armé de construction plus ancienne montre que l'utilisation des trumeaux en $\mathrm{V}$ et des autres éléments standards préfabriqués a abouti à des économies, de $70 \%$ pour le béton armé, et de $57 \%$ pour l'acier des armatures ${ }^{47}$. Étant donné l'accent porté, de manière unanime, sur la dimension économique des rotondes, celle-ci ne fait pas de doute. Remarquons toutefois que, comme source d'économies, sont systématiquement mentionnés les types de structures employées ainsi que les méthodes de mise en œuvre et l'organisation des chantiers. La contribution effective de l'étude économétrique menée en amont, au stade de la conception, est rarement mise en avant. Il est de ce fait impossible de déterminer sa réelle efficacité sur la réduction du coût de réalisation des remises, notamment en comparaison avec celles, rectangulaires, qui ont été édifiées en même temps dans les régions est et nord de la SNCF.

46- Il s'agit ici de la suppression des opérations de boisage et de coffrage pour la mise en œuvre des façades. Celle des voûtes, en revanche, nécessita la réalisations de coffrages mobiles sophistiqués...

47- Voir: La Technique des travaux, op. cit., p. 341. 


\section{Annexe}

Reconstruction des rotondes SNCF : dates, type, configuration et état actuel

\begin{tabular}{|l|c|c|r}
\hline \multicolumn{1}{|c|}{ Dépôt SNCF } & $\begin{array}{c}\text { Conception- } \\
\text { Réalisation }\end{array}$ & Type & $\begin{array}{r}\text { Nomb } \\
\text { de voi }\end{array}$ \\
\hline Avignon & $1944-1946$ & Prototype & 36 \\
\hline Béthune & $1945-1948$ & $\mathrm{G}$ & 40 \\
\hline Cambrai & $1946-1948$ & Spécial & 9 \\
\hline Chalindrey & $1947-1950$ & $\mathrm{~Pa}$ & 37 \\
\hline Creil & $1946-1948$ & $\mathrm{P}$ & 36 \\
\hline Fives-Lille & $1945-1952$ & $\mathrm{P}$ & 40 \\
\hline Hirson & $1946-1948$ & $\mathrm{P}$ & 34 \\
\hline Laon & $1946-1948$ & $\mathrm{P}$ & 36 \\
\hline $\begin{array}{l}\text { Longueau } \\
\text { (rotonde 1) }\end{array}$ & $1945-1947$ & $\mathrm{P}$ & 22 \\
\hline $\begin{array}{l}\text { Longueau } \\
\text { (rotonde 2) }\end{array}$ & $1946-1948$ & $\mathrm{G}$ & 23 \\
\hline Mantes & $1946-1947$ & $\mathrm{P}$ & 23 \\
\hline Metz & $1946-1948$ & $\mathrm{P}$ & 29 \\
\hline Noisy-le-Sec & $1946-1950$ & $\mathrm{G}$ modifié & 46 \\
\hline Poitiers & $1947-1949$ & $\mathrm{P}$ & 12 \\
\hline Sarreguemines & $1948-1952$ & $\mathrm{~Pa}$ & 31 \\
\hline Somain & $1946-1948$ & $\mathrm{G}$ & 36 \\
\hline Tourcoing & $1946-1952$ & $\mathrm{~Pa}$ Spécial & 21 \\
\hline Valenciennes & $1945-1948$ & $\mathrm{P}$ & 30 \\
\hline $\begin{array}{l}\text { Villeneuve-Saint- } \\
\text { Georges }\end{array}$ & $1946-1948$ & $\mathrm{P}$ & 32 \\
\hline
\end{tabular}

